\title{
Coaxial Anisotropy of Cortical Point Spread in Human Visual Areas
}

\author{
Soo Hyun Park, ${ }^{1 \star}$ Kuwook Cha, ${ }^{2 \star}$ and Sang-Hun Lee ${ }^{1,2}$ \\ ${ }^{1}$ Interdisciplinary Program in Neuroscience and ${ }^{2}$ Department of Brain and Cognitive Sciences, Seoul National University, Seoul 151-742, Republic of Korea
}

\begin{abstract}
A focal stimulus triggers neural activity that spreads to cortical regions far beyond the stimulation site, creating a so-called "cortical point spread" (CPS). Animal studies found that V1 neurons possess lateral connections with neighboring neurons that prefer similar orientations and to neurons representing visuotopic regions that are constrained by their preferred orientation axis. Although various roles in visual processing are proposed for this anatomical anisotropy of lateral connections, evidence for a corresponding "functional" anisotropy in CPS is lacking or inconsistent in animal studies and absent in humans. To explore functional anisotropy, we inspected axial constraints on CPS in human visual cortex using functional magnetic resonance imaging. We defined receptive fields (RFs) of unit gray matter volumes and delineated the spatial extents of CPS in visuotopic space. The CPS triggered by foveal stimuli exhibited coaxial anisotropy with larger spatial extents along the axis of stimulus orientation. Furthermore, the spatial extents of CPS along the coaxial direction increased with an increasing similarity of local sites to the CPS-inducing stimulus in orientation preference. From CPS driven by multifocal stimuli, the coaxially biased spread was also found in cortical regions in the periphery, albeit reduced in degree, and was invariant to a varying degree of radial relationship between stimuli and RF positions of local sites, rejecting radial bias as an origin of coaxial anisotropy. Our findings provide a bridge between the anatomical anisotropy seen in animal visual cortex and a possible network property supporting spatial contextual effects in human visual perception.
\end{abstract}

\section{Introduction}

A focal visual stimulus can trigger activity that spreads to an extended cortical region in which the classical receptive fields (RFs) of neurons are well outside the stimulated region (McIlwain, 1975). This phenomenon is known as "cortical point spread" (CPS). The lateral projections of the primary visual cortex are proposed as an anatomical medium for CPS based on close matches in spatial extent (Gilbert and Wiesel, 1983; Grinvald et al., 1994) and propagation velocity (Bringuier et al., 1999; Girard et al., 2001). In some species, the lateral connections in the superficial layers of V1 preferentially link columns of neurons whose RF positions and orientation preferences are aligned to a single line in visuotopic space. In this regard, the connections are biased around a visuotopic axis (Fitzpatrick, 1996; Bosking et al., 1997; Sincich and Blasdel, 2001; Chisum et al., 2003).

\footnotetext{
Received May 18, 2012; revised 0ct. 30, 2012; accepted Nov. 27, 2012.

Author contributions: S.H.P., K.C., and S.-H.L. designed research; S.H.P., K.C., and S.-H.L. performed research; S.H.P., K.C., and S.-H.L. analyzed data; S.H.P. and S.-H.L. wrote the paper.

This work was supported by the World Class University program through the National Research Foundation of Korea funded by the Ministry of Education, Science and Technology Grant R31-10089 to S.-H.L., the National Research Foundation of Korea Grant 2008-2005752 (funded by the Korea government) to S.-H.L., and in part by the National Institutes of Health Grant R01-EY016752 to S.-H.L. Some of these data were acquired while S.-H.L. was a visiting scholar at New York University. We thank Randolph Blake and David J. Heeger for the comments on earlier versions of the manuscript.

*S.H.P. and K.C. contributed equally to this work.

The authors declare no competing financial interests.

Correspondence should be addressed to Dr. Sang-Hun Lee, Department of Brain and Cognitive Sciences, Seoul National University, 1 Gwanak-ro, Gwanak-gu, Seoul 151-742, Republic of Korea. E-mail: visions!@snu.ac.kr.

K. Cha's present address: Integrated Program in Neuroscience, McGill University, 3801 University Street, Montreal, QC H3A 2B4, Canada.

DOI:10.1523/JNEUROSCI.2404-12.2013

Copyright $\odot 2013$ the authors $\quad 0270-6474 / 13 / 331143-14 \$ 15.00 / 0$
}

This presence of "coaxial" anisotropy in V1 lateral connections, despite substantial differences across species (Douglas and Martin, 2004), raises the question of whether CPS in V1 also exhibits an axial anisotropy biased toward the orientation axis of the stimulus that originally triggered the CPS. However, there has been only one study that has investigated the axial anisotropy of CPS (Chisum et al., 2003). Furthermore, in that study, it was inconclusive whether coaxial anisotropy was present in CPS because only an insignificant degree of anisotropy was indirectly found in a highly limited condition.

In addition, although psychophysical studies have ascribed various roles in visual processing for coaxial anisotropy in lateral connections (Hess and Field, 1999; Polat, 1999; Wilson et al., 2001; Pillow and Rubin, 2002; Series et al., 2002; Greenwood et al., 2010), neither functional nor anatomical coaxial anisotropy of CPS has been examined in human visual cortex. The characterization of axial anisotropy of CPS in human visual cortex could provide a crucial step toward bridging the interspecies and interdomain gaps between the anatomical anisotropy seen in animal visual cortex and the spatial contextual effects in human visual perception.

Here, we examined the functional anisotropy of CPS in early human visual areas by obtaining the following series of functional magnetic resonance imaging (fMRI) measurements. We first defined the RF positions at a fine resolution and the stimulus preferences for the individual voxels of V1, V2, and V3. We then measured responses of those voxels to a single static grating presented at the fixation and to multiple dynamic gratings that moved across a large visual field while varying the orientations of the gratings. The combined analyses of these measurements sup- 
ported the presence of coaxially anisotropic CPS by establishing the following set of findings. The activity triggered by the foveal grating spreads anisotropically on the cortical surface, such that the angle of anisotropy was aligned with the meridian defined by the grating orientation, with a degree of coaxial anisotropy exaggerated for the CPS constructed by the subset of voxels preferring the grating orientation. Using multiple gratings that triggered traveling waves of CPS, we demonstrated that the coaxial anisotropy decreased with an increasing eccentricity while being invariant to radiality.

\section{Materials and Methods}

\section{Subjects and scanning sessions}

Eight subjects (two of the authors and six naive subjects, four members of each sex, 20-28 years old) participated in the experiments. All subjects had normal or corrected-to-normal vision and provided written informed consent before participating in experiments. Three subjects participated in experiments at New York University (NYU) and five at Seoul National University (SNU). Experiments conducted at NYU were in compliance with the safety guidelines for MRI research and approved by the University Committee on Activities Involving Human Subjects at NYU. The SNU Institutional Review Board approved the experiments at SNU. Each subject participated in multiple scanning sessions: one session for acquiring a high-resolution volume anatomy, one session of conventional retinotopy experiments, one session of population receptive field $(p R F)$ mapping experiments, two sessions of static CPS experiments with unifocal triggers, and one session of traveling CPS experiments with multifocal triggers. Each fMRI scanning session included 10 or 11 functional runs.

\section{Visual stimulus presentation}

At NYU, visual stimuli were presented using a LCD projector (Eiki LCXG100; Eiki) with a pixel resolution of $1024 \times 768$ and a refresh rate of 60 $\mathrm{Hz}$. Subjects viewed images on a rear projection screen placed inside the bore of the magnet at a distance of $57 \mathrm{~cm}$, yielding a field of view of $29^{\circ} \times$ $22^{\circ}$. This device was calibrated using a Photo Research PR650 SpectraColorimeter to achieve a linear gamma. At SNU, visual stimuli were presented using a LCD projector (Canon XEED SX60; Canon) with a pixel resolution of $1400 \times 1050$ and a refresh rate of $60 \mathrm{~Hz}$. Subjects viewed the images through an angled mirror on a rear projection screen placed inside the bore of the magnet at a distance of $87 \mathrm{~cm}$, yielding a field of view of $22^{\circ} \times 17^{\circ}$. This display was calibrated using a Minolta luminance meter LS-100 (Konica Minolta Holdings) to achieve a linear gamma. Stimuli were generated using Matlab (MathWorks) and MGL (available at http://justingardner.net/mgl) on a Macintosh computer.

\section{Conventional retinotopic mapping and definition of visual areas of interest}

Retinotopic mapping of visual field eccentricity and polar angle for each subject was achieved using a conventional "phase-encoding" method (Engel et al., 1994; Sereno et al., 1995; Engel et al., 1997). High-contrast checkerboard stimuli were presented as rotating wedges, expanding/contracting rings, or bars at the horizontal/vertical meridian. Each run consisted of 10 cycles, and the first cycle of fMRI responses was discarded. A scanning session was composed of two runs of the ring stimulus (one expanding and one contracting), four runs of the wedge stimulus (two clockwise and two counterclockwise), and one run of the meridian bar stimulus (horizontal and vertical meridian stimulation were alternated between cycles). The temporal phases of the fMRI responses were visualized on flattened representations of the cortical surface, which were segmented from a high-resolution 3D volume rendering of each subject using Freesurfer (Dale et al., 1999; Fischl et al., 1999) (http://surfer.nmr. mgh.harvard.edu). Visual areas V1, V2, and V3 were defined manually following published conventions (Larsson and Heeger, 2006).

\section{Population receptive field mapping experiment}

To ensure precise receptive field location estimates for individual gray matter unit volumes (voxels), a modified version of the model-driven retinotopy method was designed (Dumoulin and Wandell, 2008). The subjects were presented with a dynamic dartboard pattern within a bar aperture that drifted smoothly and periodically within a virtual aperture of a large circle $\left(10^{\circ}\right.$ in radius at NYU and $8^{\circ}$ at SNU) while fixating on the center of the screen. The pedestal dartboard pattern was generated as a product of two equiangular spirals with pitches that changed every $1.5 \mathrm{~s}$ to effectively evoke cortical activity by dynamically stimulating a wide range of orientation-tuned and spatial frequency-tuned neural populations in the visual cortex. The spatial and temporal lengths of a single sweep of the bar drift were $27^{\circ}$ and $27 \mathrm{~s}$, respectively, which yielded a drifting velocity of $1 \%$ s. This spatiotemporal structure resulted in a duty cycle of $3 \mathrm{~s}$ on stimulation and $24 \mathrm{~s}$ off stimulation periods at any given pixel within the viewing aperture. The width of the bar aperture was $3^{\circ}$, and the contrast of the dartboard pattern was ramped smoothly at the edges with a cumulative Gaussian function $\left(\sigma=0.1^{\circ}\right)$ to minimize second-order spatial features and transient signals. The bar aperture drifted perpendicular to its long axis during a scan run and rotated from $0^{\circ}$ to $315^{\circ}$ with $45^{\circ}$ steps in a polar angle dimension over the eight scan runs.

A hemodynamic impulse response function (HIRF) was estimated with two separate scan runs (one at the beginning and the other at the end of a session), during which the subjects viewed the dartboard pattern, which was identical to the pedestal stimulus in the $p R F$ mapping scan runs, as a whole within the circle aperture $\left(10^{\circ}\right.$ in radius at NYU and $8^{\circ}$ at SNU). To preclude any potential errors in HIRF estimation resulting from imperfect temporal linearity of the fMRI responses, the stimulus was turned on for $3 \mathrm{~s}$ and off for $24 \mathrm{~s}$ so that the on/off durations at any given pixel were matched between the HIRF and the $p R F$ mapping scan runs. The contrast was temporally smoothed both at the onset and the offset of stimulation to minimize transient neural responses that cannot be captured by our simplified linear model of neural responses.

In both the $p R F$ mapping and HIRF runs, subjects were asked to perform a fixation task to maintain overall attention at a constant level and eye position fixed at the center of the screen. In the fixation task, subjects pressed a button whenever two diametrically opposed small $\left(0.07^{\circ}\right.$ diameter) dots, which traveled along the annulus $\left(0.4^{\circ}\right.$ in diameter $)$ trajectory around the fixation, changed their direction of rotation. To make rotation reversals frequent but unpredictable, the durations of continuous rotations were stochastically sampled using a Poisson distribution with a mean of $2 \mathrm{~s}$.

\section{Static CPS experiment with unifocal triggers}

To generate CPS, we presented oriented $\left(0^{\circ}, 90^{\circ}, 45^{\circ}\right.$, or $\left.135^{\circ}\right)$ sinusoidal gratings at the fixation point (see Fig. $2 A$ ). We will refer to these CPSinducing stimuli as "triggers" from here on. The static CPS experiment consisted of two separate daily scanning sessions. The orientations of the triggers were $0^{\circ}$ and $90^{\circ}$ in the "cardinal orientation" session and $45^{\circ}$ and $135^{\circ}$ in the "oblique orientation" session. Each session consisted of six "CPS triggering $(\mathrm{C})$ " runs, three "orientation preference mapping $(\mathrm{O})$ " runs, and two "HIRF estimation $(\mathrm{H})$ " runs. The runs were conducted in the following fixed order: $\mathrm{H}, \mathrm{O}, \mathrm{C}, \mathrm{C}, \mathrm{O}, \mathrm{C}, \mathrm{C}, \mathrm{O}, \mathrm{C}, \mathrm{C}$, and $\mathrm{H}$.

In the CPS triggering runs, a block design was used, in which a trial consisted of a $9 \mathrm{~s}(=1.5 \mathrm{~s} \times 6 \mathrm{MRI}$ image frames $)$ period of stimulus on and a $15 \mathrm{~s}(=1.5 \mathrm{~s} \times 10 \mathrm{MRI}$ image frames) period of stimulus off (Fig. $2 B$ ). A single run consisted of 10 trials, with fMRI responses from the first trial discarded from the analysis. In total, six CPS triggering runs per session yielded 18 trials for each stimulus orientation. To minimize stimulus adaptation, a trigger was switched on and off every $1.5 \mathrm{~s}$ periodically during the stimulus-on period. The trigger stimulus was a $0.8^{\circ}$ radius circular patch with the contrast of the edges smoothed using a cumulative Gaussian function $\left(\mu=0.8^{\circ} ; \sigma=0.2^{\circ}\right)$. The central $\left(0.3^{\circ}\right.$ diameter $)$ portion of the grating was masked with a bull's-eye fixation. One of the three (two oriented gratings and one bandpass-filtered random-dot stimulus) types of triggers was shown in any given block, and the order of presentation of the triggers in a given run was counterbalanced across runs. The spatial phase of gratings was randomized for every presentation. The spatial frequency of a grating averaged 2 cycles $/{ }^{\circ}$ and ranged from 1.8 to 2.2 cycles $/{ }^{\circ}$. During the stimulus-on period, we slightly altered the spatial frequency of triggers across trials and asked subjects to report whether the stimulus frequency was higher or lower than the 
average. To maintain constant task difficulty, the amount of alteration in the spatial frequency was continuously adjusted in small steps $(0.05 \mathrm{cy}-$ cles $/^{\circ}$ ) based on performance. During the stimulus-off period, subjects performed a fixation task that entailed indicating the thickness of the outer circle of the bull's-eye with a button press every $1.5 \mathrm{~s}$.

In the orientation preference mapping runs, the stimulus orientation was alternated in blocks of $12 \mathrm{~s}$, in which the grating was switched on and off every $1.5 \mathrm{~s}$ (see Fig. $2 C$ ). We slightly varied the orientation of the stimuli $\left(0.15^{\circ}-0.45^{\circ}\right.$ offset $)$ around a reference orientation in a given period $\left(0^{\circ}\right.$ or $90^{\circ}$ for the cardinal session and $45^{\circ}$ or $135^{\circ}$ for the oblique session) across trials and asked subjects to indicate whether the grating orientation was tilted clockwise or counterclockwise relative to a reference. To maintain constant task difficulty, the amount of the orientation offset was adjusted in small steps $\left(0.15^{\circ}\right)$ based on performance after each run.

In the HIRF estimation runs, a large circular disk of high contrast dartboards was split into an inner $\left(4^{\circ}\right.$ radius) and outer $\left(4^{\circ}-8^{\circ}\right.$ or $10^{\circ}$ radius) annulus. The inner annulus was used in the first run, and the outer was used in the second run. Apart from stimulus size, the temporal structure and stimulus parameters were identical to those used in the CPS triggering runs.

\section{Traveling CPS experiment with multifocal triggers}

For each run of the traveling CPS experiment, a vertical or horizontal stripe of Gabor patches $\left(\sigma=0.42^{\circ}\right)$ drifted periodically across the entire visual field in a single direction perpendicular to the orientation of the stripe while subjects fixated on the center of the display (see Fig. 5A). To minimize potential second-order interaction effects between spatiotemporally neighboring local Gabors in the multifocal display, a series of constraints was imposed on the spatiotemporal layout of the Gabor patches. First, in the spatial domain, Gabors were separated by $3^{\circ}$ in a given vertical or horizontal stripe. Second, in the temporal domain, a stripe of Gabors remained at a given position for $0.75 \mathrm{~s}$ and was then followed by another stripe in a new position that was shifted by $0.75^{\circ}$. Third, the entire set of Gabors within a stripe were shifted (vertically within a vertical stripe or horizontally within a horizontal stripe) by $1.5^{\circ}$ in terms of relative position at every step of shift so that any local Gabor was not followed nor preceded by another Gabor that is aligned with the shifting direction of a stripe. Fourth, we randomly varied the seed spatial phases of Gabor elements composing a stripe to prevent collinear arrangements of luminance lobes with the same polarity. In addition, we also altered the spatial phases of Gabors by $90^{\circ}$ at every step of a shift. To provide an effective trigger at any given eccentricity, we assigned spatial frequency values to individual Gabors based on their eccentricity values by applying the following logarithmic function:

$$
S F_{i}=S F_{\text {fovea }}-5 / 6 * \log \varepsilon_{i},
$$

where $S F_{i}$ is the spatial frequency of Gabor element $i, S F_{\text {fovea }}$ is the spatial frequency at the fovea $\left(3\right.$ cycles $\left.^{\circ}\right)$, and $\varepsilon_{i}$ is the eccentricity of Gabor element $i$. As a result, the spatial frequency ranged from $1 \mathrm{cycle} /{ }^{\circ}$ at an eccentricity of $10^{\circ}$ to 3 cycles $/{ }^{\circ}$ at the fovea. In a given run, the orientation of Gabor elements alternated between collinear and orthogonal to the shifting direction of the stripe between cycles. Each run consisted of nine cycles, including one dummy cycle at the beginning, four cycles with the coaxial conditions, and four cycles with the ortho-axial conditions. A session consisted of eight runs in total, two runs for each of the four different shifting directions of the stripe (rightward, upward, leftward, and downward), resulting in a total of 32 cycles $(4$ cycles $\times 8$ runs) for both the coaxial and ortho-axial conditions. At the start and end of each session, there were two HIRF estimation runs, during which the whole visual field of observation $\left(10^{\circ}\right.$ or $8^{\circ}$ in radius) was stimulated with impulses of high contrast bandpass $\left(1-3\right.$ cycles $\left./{ }^{\circ}\right)$ filtered images of random dots. The spatial and temporal structure of stimulation was the same as the HIRF runs used in the $p R F$ mapping experiment. Subjects also performed the same fixation task as in the $p R F$ mapping experiment.

\section{MRI acquisition}

MRI data were acquired at NYU using a Siemens 3T Allegra head-only scanner with a transmit head coil (NM-011; NOVA Medical) and a four- channel phased array receive coil (NMSC-021; NOVA Medical). The pulse sequence parameters for the main experimental ( $p R F$ and CPS estimation) scan sessions were as follows: 24 slices, $1.5 \mathrm{~s}$ repetition time, $30 \mathrm{~ms}$ echo time, $75^{\circ}$ flip angle, $2 \mathrm{~mm} \times 2 \mathrm{~mm} \times 2 \mathrm{~mm}$ voxel size, $120 \times$ 80 matrix size. The parameters for the conventional retinotopy scan sessions were as follows: 16 slices, $1.2 \mathrm{~s}$ repetition time, $30 \mathrm{~ms}$ echo time, $75^{\circ}$ flip angle, $3 \mathrm{~mm} \times 3 \mathrm{~mm} \times 3 \mathrm{~mm}$ voxel size, $64 \times 64$ matrix size. Oblique slices were positioned perpendicular to the calcarine sulcus to cover the target visual areas (V1, V2, and V3). At the beginning of each experimental session, T1-weighted (MPRAGE) anatomical volume images were acquired from the same slices used for the functional scans at higher in-plane voxel resolutions $(1.5 \mathrm{~mm} \times 1.5 \mathrm{~mm} \times 3 \mathrm{~mm}$ for the main experimental scans and $1 \mathrm{~mm} \times 1 \mathrm{~mm} \times 3 \mathrm{~mm}$ for the conventional retinotopy scans).

At SNU, MRI data were acquired using a Siemens 3T Magnetom Trio scanner with a 12-channel head coil. The parameters for the conventional retinotopy scan sessions were as follows: 30 slices, $2.7 \mathrm{~s}$ repetition time, $40 \mathrm{~ms}$ echo time, $77^{\circ}$ flip angle, $2 \mathrm{~mm} \times 2 \mathrm{~mm} \times 2 \mathrm{~mm}$ voxel size, $104 \times 104$ matrix size. For the other experiments, a zoomed EPI protocol was used with oversampling in the phase encoding direction to achieve the desired in-plane resolution and to remove any fold-in signal from outside the field of view. The pulse sequence parameters for the zoomed EPI were as follows: 18 slices with $0.5 \mathrm{~mm}$ gaps, $1.5 \mathrm{~s}$ repetition time, 37 $\mathrm{ms}$ echo time, $75^{\circ}$ flip angle, $2 \mathrm{~mm} \times 2 \mathrm{~mm} \times 2 \mathrm{~mm}$ voxel size, $75 \%$ phase oversampling, $128 \times 32$ matrix size. Slices were located almost parallel to the calcarine sulcus. The in-plane resolution of T1-weighted (MPRAGE) anatomical volume images was $1 \mathrm{~mm} \times 1 \mathrm{~mm} \times 2.5 \mathrm{~mm}$.

\section{MRI data preprocessing}

For each brain, the T1-weighted in-plane images were aligned to the high-resolution anatomical volume using either an automated robust image registration algorithm (Nestares and Heeger, 2000) for the NYU dataset or a mutual information algorithm provided by statistical parametric mapping software (SPM8; http://www.fil.ion.ucl.ac.uk/spm; Wellcome Department of Cognitive Neurology, London, United Kingdom) for the SNU dataset. Motion artifacts within and across functional runs were corrected using a standard procedure for motion compensation (Friston et al., 1996; Jenkinson et al., 2002). The functional runs were aligned to the high-resolution volume anatomy using a transformation matrix obtained from the alignment of the T1-weighted in-plane images to the high-resolution volume anatomy. For each scan run, the raw time series of fMRI responses measured in individual voxels were high-pass filtered with a cutoff frequency, which was the reciprocal of the stimulus on/off cycle duration $[0.037 \mathrm{~Hz}$ (27 s cycle) for the conventional retinotopy mapping, the $p R F$ mapping, and the traveling CPS experiments; $0.042 \mathrm{~Hz}$ (24 s cycle) for the static CPS experiments], and then converted into percentage signal changes by dividing the time series of each voxel by its mean intensity. Data from the first cycle of each functional run were discarded to avoid artifacts originating from transient magnetic saturation and to allow hemodynamic responses to reach a steady state.

\section{Selection of voxels for analysis}

A precise mapping of the $p R F$ s of individual voxels is essential for the valid estimation of CPS in visuotopic space, so we applied conservative criteria when selecting valid voxels for further analysis. Voxels that include large blood vessels near the pial surface were discarded from analysis because their fMRI signals are decoupled from neural activity both in the spatial domain (mislocalization errors) (Olman et al., 2007; Polimeni et al., 2010) and in the temporal domain (delayed time series) (Silva et al., 2000). Temporal decoupling could lead to systematic errors in $p R F$ and CPS estimation because we estimated $p R F$ and CPS in visuotopic space from the time courses of fMRI responses. We identified and discarded "decoupled" voxels by two methods. First, we computed the 3D volume coordinates of individual voxels in the functional scans and then discarded any voxels whose volume extended to the outside of the gray matter based on the known average gray matter thickness of human visual cortex $(3 \mathrm{~mm})$ and the dimensions of a functional voxel size (2 $\mathrm{mm} \times 2 \mathrm{~mm} \times 2 \mathrm{~mm}$ ). As a result, the voxels that survived this restriction rule were those whose volume centers were below the gray matter 
depth of $1.5 \mathrm{~mm}$. The second criterion was developed based on the fact that "blood-vessel-clamping" voxels give low intensities in gradient echo $\mathrm{T} 2{ }^{*}$ images and give unusually high amplitudes of fMRI response. Thus, those voxels whose $\mathrm{T}^{\star}$ values were in the lowest $10 \%$ or whose fMRI amplitudes were in the highest 10\% were discarded (Shmuel et al., 2007).

\section{Population receptive field mapping of voxels}

The $p R F$ s of individual voxels were modeled by an isotropic 2D Gaussian function in visuotopic space (Dumoulin and Wandell, 2008). First, neural responses were predicted by convolving the spatiotemporal matrix of the drifting bar stimulus $\mathrm{s}(x, y, t)$ with the $2 \mathrm{D}$ gain function of RF:

$$
g(x, y)=a e^{-\left(\left(x-x_{0}\right)^{2} / 2 \sigma^{2}+\left(y-y_{0}\right)^{2} / 2 \sigma^{2}\right)},
$$

where $x_{0}$ and $y_{0}$ are the $x$ and $y$ coordinates of the $p R F$ center, and $\sigma$ and $a$ are the SD and amplitude of the isotropic 2D Gaussian function, respectively. fMRI responses were then predicted by convolving the predicted neural responses with HIRF, which was estimated separately for individual subjects and visual areas by fitting a two- $\gamma$ model of hemodynamic response (Glover, 1999) to the data from the HIRF estimation runs. Finally, for each voxel, the four free model parameters $\left(x_{0}, y_{0}, \sigma\right.$, and a) were estimated by fitting the predicted fMRI responses to the observed fMRI responses in all of the eight drifting bar runs simultaneously. The goodness of fit of the model predictions $\left(r^{2}\right)$ was computed for each voxel with the amount of variance in the raw fMRI responses accounted for by the predicted fMRI responses and was further used for valid voxel selection.

In addition to obtaining an accurate map of $p R F$ s for voxels of interest, the alignment between the $p R F$ and CPS sessions was another critical step in acquiring valid CPS estimations. The coregistration between the two sessions was accomplished as follows. First, all of the functional (F) sessions were separately aligned to one single reference $(\mathrm{V})$, the subject's own high-resolution 3D volume anatomy, by identifying best-matching transformation matrices. Based on this set of F-to- $\mathrm{V}$ transformation matrices, we identified a voxel in the CPS session that corresponded to a voxel in the $p R F$ session by first transforming a voxel coordinate in the $p R F$ session into a coordinate in the reference volume using the $\mathrm{F}_{p R F^{-}}$ to- $\mathrm{V}$ matrix and then by transforming that reference volume coordinate into a voxel coordinate in the CPS session using the inverse of the $\mathrm{F}_{\mathrm{CPS}^{-}}$ to- $\mathrm{V}$ matrix. Note that, because all seed voxels for further analyses were defined in the $p R F$ sessions, the starting points for all of these transformations were voxels in the $p R F$ sessions.

It is reasonable to assume a degree of imperfectness in coaligning the functional scan slices between the $p R F$ session and the main experimental sessions. To assess the impact of potential coalignment errors on the accuracy of $p R F$ estimation, we calculated the amount of $p R F$ estimation errors that would have been caused by the maximum possible amount of coalignment errors. By applying the "worst-case" scenario, we artificially shifted the 3D anatomical coordinates of the entire V1 voxels of one sample experimental session by a unit size $(2 \mathrm{~mm})$ on each of the three dimensions, and calculated the resulting offsets from the original data in terms of polar angle position in visuotopic space, a critical dimension for accurate anisotropy estimation. Note that this "worst-case" scenario exercised here is highly unlikely to occur in a real situation because such misalignment as large as the unit voxel dimension would be readily detectible (e.g., gray matter voxels placed in the skull) and calls for another round of iterative alignments until a sufficiently small degree of error (typically well within one voxel size) is obtained.

\section{Data analysis for static CPS}

Preprocessing of $f M R I$ time series. For each voxel, time series in percentage signal were chopped and stacked on a trial-by-trial basis by taking 18 time frames, including the two frames of the prestimulus period. The baseline of time series from individual trials was adjusted to zero, which was defined as the averaged responses of the initial two prestimulation frames. The "peak" time frames (Fig. $3 A$, shaded region) of those 18frame time series were defined by identifying three consecutive frames where average responses were maximally varied across individual trials of times series for all the voxels within each visual area ROI. The definition of peak frames based on variance allowed for the capture of peak re- sponses both in the positive side (voxels whose $p R F$ s are near the fovea) and in the negative side (voxels whose $p R F$ s are away from the fovea). For each voxel and each trial, the amplitude was computed by averaging responses within those peak frames.

Trial sorting. For the analysis of axial anisotropy, trials were sorted into the coaxial condition when the absolute offset of the angular position of a given voxel's $p R F$ from the trigger orientation is within $22.5^{\circ}$, or into the ortho-axial condition when the offset is $>67.5^{\circ}$. Because the trigger orientation varied across trials, the identity of a given voxel as either coaxial or ortho-axial was determined on a trial-by-trial basis. This method of sorting resulted in the same group of voxels for both conditions that differed only by the orientation of the trigger to which they responded.

$f M R I$ point spread function analysis. The response amplitudes of individual voxels were sorted according to their $p R F$ eccentricity and derived the fMRI point spread function ( $f P S F$ ) (Fig. 3B):

$$
f P S F=\overline{P R}(\varepsilon) ; \varepsilon_{v o x=i}=\sqrt{x_{i}^{2}+y_{i}^{2}},
$$

where $\overline{P R}$ is the response amplitude of fMRI time series averaged across trials and $x_{i}$ and $y_{i}$ are Cartesian coordinates of the $p R F$ center of voxel $i$ in visuotopic space. Given the presence of positive and negative lobes of fPSFs (see Fig. $3 A, B$ ), indicating excitation at the site of direct stimulation and suppression at the peripheral sites, we fit the difference of Gaussians (DoG) function to fPSFs because the DoG function has been routinely used to describe the center-surround organization of RFs of V1 simple cells (Hawken and Parker, 1987). Because the distance metric of the DoG function is assumed to be in the scale of the cortical surface, the fPSF was rescaled by changing the distance metric from an eccentricity scale to a cortical distance (from the stimulus edge) scale by applying the canonical cortical magnification factor for human visual cortex (Horton and Hoyt, 1991):

$$
\begin{gathered}
C=17.3 \log (\varepsilon+0.75) ; \\
C_{r e l}=C-17.3 \log (0.8+0.75)=C-5.1918 .
\end{gathered}
$$

Then, voxels were binned using a sliding window of $5 \mathrm{~mm}$ width and a step size of $1 \mathrm{~mm}$. Within each bin, response amplitudes were resampled to generate 1000 bootstrap sample means using a parametric bootstrap method. For each bootstrapped sample, fPSFs were built and used to fit the DoG model:

$$
\operatorname{DoG}\left(C_{r e l}: \mu, \sigma_{E}, \sigma_{I}\right)=A \frac{1}{\sqrt{2 \pi \sigma_{E}^{2}}} e^{-\frac{\left(C_{r e l}-\mu\right)^{2}}{2 \sigma_{E}^{2}}}-A \frac{1}{\sqrt{2 \pi \sigma_{I}^{2}}} e^{-\frac{\left(C_{r e l}-\mu\right)^{2}}{2 \sigma_{I}^{2}}} .
$$

This DoG function was separately fitted to the individual $f P S F$ s for each area of each subject. For fitting observed $P P S F$, a maximum likelihood estimation method was used and for fitting bootstrapped $f P S F$, a least square estimation method was used. The initial parameter $\sigma_{I}$ was $4^{\star} \sigma_{E}$ except for data from area $\mathrm{V} 3$ of subject $\mathrm{S} 8$, which required a larger initial $\sigma_{I}\left(=6^{*} \sigma_{E}\right)$ because of the wide $f P S F$. The initial estimate for $A$ was the maximum response amplitude of $P P S F$. Using the output parameters of observed $f P S F$ fitting as initial estimates, bootstrapped $P P S F$ s were fitted. The spatial extent (Fig. 3, $E_{c}$ and $E_{o}$ ) was estimated by computing the eccentricity at which $P P S F$ was equal to 0 (i.e., the boundary of positive fMRI responses). Confidence intervals for spatial extent were estimated using the bootstrap method.

To quantify how much spatial extents differ between the two axial conditions relative to the overall size of spread, we computed an axial anisotropy index $\left(A I_{A X}\right.$; Fig. $\left.3 D\right)$ as follows:

$$
A I_{A X}=\frac{\left(E_{c}-E_{o}\right)}{\left(E_{c}+E_{o}\right)}
$$

This index ranges from -1 to 1 , being positive (negative) when $P P S F$ is more (less) extended in coaxial condition than in ortho-axial condition. The significance of $A I_{A X}$ was tested across subjects using the Wilcoxon signed rank test. 
We also repeated the above analysis on the same dataset with eccentricity bins that were defined to be equal sizes in visuotopic (visual angle) scale. In this alternative analysis, $P P S F$ was defined by applying a sliding window with $0.8^{\circ}$ in width and $0.2^{\circ}$ in step size. The remaining procedure was identical to that in the analysis with the cortical distance metric, except that the $C_{r e l}$ in Eq.5 was replaced with the visual-angle eccentricities relative to the stimulus edge $\left(0.8^{\circ}\right)$.

Indexing orientation preferences. The static CPS estimation sessions included three orientation preference mapping runs (see Fig. $2 C$ ). The orientation preference was defined by the following steps. First, the time series of each run were cut into $12 \mathrm{~s}$ (eight frames) time series locked to stimulus onsets, and then sorted into the two orientation conditions (except for two junk trials in the first cycle), yielding 27 trials for each of the orientation conditions. Second, individual fMRI responses between the fifth and eighth frames in each trial were extracted to determine the distributions of frame-by-frame fMRI responses (one for each of the orientation conditions). Third, the binary orientation preference index (OPI; see Fig. 4A) was computed from these two distributions by dividing the difference between the means of the distributions $\left(\bar{R}_{1}\right.$ and $\left.\bar{R}_{2}\right)$ by their pooled SDs:

$$
\begin{gathered}
\text { OPI }=\left(\bar{R}_{1}-\bar{R}_{2}\right) / S_{\text {pooled }} ; \\
S_{\text {pooled }}=\sqrt{\frac{\left(N_{1}-1\right) S_{1}^{2}+\left(N_{2}-1\right) S_{2}^{2}}{N_{1}+N_{2}-2}},
\end{gathered}
$$

where $S_{1}$ and $S_{2}$ are the SDs of the two distributions and $N_{1}$ and $N_{2}$ are the number of responses for the two distributions $\left(N_{1}=N_{2}=135\right)$. The sign of OPI indicates the direction of preference, and its absolute value represents the degree of binary orientation preference.

Orientation dependency of axial anisotropy analysis. For the analysis of the dependency of axial anisotropy on orientation preference, the V1 data in the coaxial trials were merged across all subjects to secure a substantial number of voxels (4137 voxels) for analysis. Before the data were merged the raw response amplitudes were divided by the average response of voxels that were directly stimulated by the trigger (i.e., voxels with $p R F$ s that were within $0.8^{\circ}$ of eccentricity). This allowed the normalization of data from different subjects for possible inherent differences in BOLD response amplitudes across individuals.

Based on the distribution of OPIs, voxels were further categorized into three different preference groups. First, voxels that yielded negative OPI values were categorized into an unmatched group (1326 voxels; Fig. 4A, white bars). Next, the remaining voxels whose preferences matched the trigger stimulus were further split at their median OPI value (0.3544) into either weak or strong preference matched groups (1405 voxels for each group; Fig. $4 A$, gray and black bars, respectively). We then derived the fPSFs for each of those preference groups and compared their spatial extents. For statistical analysis of the between-group differences in spatial extent, we generated 1000 parametric bootstrap samples of $f P S F$ s for each of the preference groups and then obtained 1000 difference values in spatial extent between the two groups. The $p$ value for the positive (or negative) difference between the two groups was then computed as the percentage of the bootstrap-sampled differences below (or above) zero.

\section{Data analysis for traveling CPS}

To estimate the width and amplitude of traveling CPS, we first estimated times to peak fMRI activity for individual voxels using cyclic cosine functions and then fitted Gaussian functions to fMRI time series data whose time axis is defined as time relative to the time to peak activity. The detailed procedures were as follows.

Estimation of the time to peak fMRI activity. The time to peak fMRI activity was estimated by fitting two cyclic cosine (von Mises) functions simultaneously to the fMRI time series from the coaxial and coorientation conditions. First, in a given run (e.g., a run where the vertical stripe was moving rightward), the original time series was cut into 36frame (54 s) trials containing two consecutive cycles, one cycle of coaxial triggers ( 18 frames) and one cycle of ortho-axial triggers (18 frames), and these were then averaged across trials. The same process was then carried out for the data from the run with the same stripe moving in the opposite direction (e.g., a run where the vertical stripe was moving leftward). The two cyclic cosine functions were then fitted simultaneously to this pair of stacked time series obtained from the pair of runs with the stripes of the same orientation moving in opposite directions using the least square estimation method. Specifically, the function that was used is described as follows:

$$
\begin{aligned}
& R_{1}=a_{c} * e^{b_{c} * \cos \left(x-p_{1}\right)}+a_{o} * e^{b_{o} * \cos \left(x-\left(p_{1}+\pi\right)\right)}+m \\
& R_{2}=a_{c} * e^{b_{c} * \cos \left(x-p_{2}\right)}+a_{o} * e^{b_{o} * \cos \left(x-\left(p_{2}+\pi\right)\right)}+m,
\end{aligned}
$$

where $R_{1}$ and $R_{2}$ are fMRI time series from the two moving direction runs, $a_{c}$ and $a_{o}$ are the amplitudes for the two axial conditions, and $m$ is the baseline fMRI response. Based on the assumption that CPS is onlydependent on the axiality of the trigger but is invariant to the shifting direction of the trigger, the same parameters $a$ and $b$ were applied to each axial condition regardless of the moving direction of the stripe (i.e., both in $R_{1}$ and $R_{2}, a_{c} / b_{c}$ were applied to the coaxial condition and $a_{o} / b_{o}$ to the ortho-axial condition). Note that the phase parameters differed by $\pi$ between the coaxial and the ortho-axial time series since the two were the same in length and made one full cycle together. Initial parameters for the phase, $p_{1}$ and $p_{2}$, were estimated from the $p R F$ coordinates of a given voxel: $x$-axis coordinates for the vertical stripe and $y$-axis coordinates for the horizontal stripe. The goodness of fit of the model predictions $\left(r^{2}\right)$ was computed for each voxel. Since the correct estimation of times to peak fMRI activity is critical for defining CPSs accurately, we applied conservative criteria for selecting valid voxels for further analysis based on the fraction of the variance that was explained by the model fit while keeping enough number of voxels in given datasets $\left(r^{2}>0.2-0.7\right)$.

Estimation of the width and amplitude of fMRI time series. The width and amplitude of CPSs were estimated in the following steps. First, based on the location parameters $\left(p_{1}\right.$ and $\left.p_{2}\right)$ of the cyclic cosine functions fit to the data, we aligned (via cyclic shifting) the raw time series of individual voxels to their own time to peak. Second, individual voxels were sorted into four nonoverlapping eccentricity bins that split the range of $1^{\circ}$ to $7.4^{\circ}$ equally (thus, bin width $=1.6^{\circ}$ ). The voxels with eccentricity $<1$ were excluded from analysis because the time series were likely to be contaminated by their responses to the fixation dot and associated fixational eye movements. Third, for each of those eccentricity bins, the time series from individual voxels are sorted into the coaxial and the orthoaxial conditions (as described in Fig. 5A) and stacked together to form a population CPS (pCPS; Fig. 5B). Fourth, we fit a Gaussian function to those pCPSs separately for the two axial conditions using the least square estimation method:

$$
\begin{gathered}
p C P S_{c}\left(t_{r e l}: \alpha_{c}, \sigma_{c}, B\right)=\alpha_{c} * e^{-\frac{t_{r e l^{2}}}{2 \sigma_{c} c^{2}}}+B ; \\
p C P S_{o}\left(t_{r e l}: \alpha_{o}, \sigma_{0}, B\right)=\alpha_{o} * e^{-\frac{t_{r e l}{ }^{2}}{2 \sigma_{0}^{2}}}+B,
\end{gathered}
$$

where $t_{r e l}$ is the time relative to peak; $\alpha_{c}$ and $\alpha_{o}$ are the amplitudes, and $\sigma_{c}$ and $\sigma_{o}$ are the sigmas for the coaxial and the ortho-axial conditions, respectively; $B$ is the baseline fMRI response, a parameter shared by the two axial conditions.

Computation of width-based and amplitude-based axial anisotropy indices. Based on the amplitude $\left(\alpha_{c}, \alpha_{\mathrm{o}}\right)$ and width $\left(\sigma_{\mathrm{c}}, \sigma_{\mathrm{o}}\right)$ parameters estimated at each eccentricity bin, the anisotropy indices based on peak amplitude $\left(A I a_{A X}\right)$ and width $\left(A I \sigma_{A X}\right)$ were computed by taking the signed difference in parameter values between the two axial condition and then dividing the difference by the sum of two values:

$$
A I \alpha_{A X}=\frac{\left(\alpha_{c}-\alpha_{o}\right)}{\left(\alpha_{c}+\alpha_{o}\right)} ; A I \sigma_{A X}=\frac{\left(\sigma_{c}-\sigma_{o}\right)}{\left(\sigma_{c}+\sigma_{o}\right)} .
$$

The significance of these anisotropy indices was tested using pairedsample $t$ test. We confirmed that the Wilcoxon signed rank tests also gave similar statistical results.

Analysis of radiality contingency of coaxial anisotropy. To examine the possible dependency of the two types of anisotropy indices on the radiality, CPSs were defined separately for the three radiality conditions (the 
radial, neutral, and ortho-radial conditions), which were defined jointly by the meridian angular position of a given voxel's $p R F$ and stimulus orientation in the following steps. First, voxels were categorized into three meridian groups according to the polar angle positions of their $p R F$ centers $\left(\theta_{R F}\right)$ : the horizontal meridian group $\left(0.8660<\left|\cos \left(\theta_{R F}\right)\right|\right)$, the oblique meridian group $\left(0.5<\left|\cos \left(\theta_{R F}\right)\right|<0.8660\right)$, and the vertical meridian group $\left(\left|\cos \left(\theta_{R F}\right)\right|<\right.$ $0.5)$. Second, for the horizontal and vertical meridian groups, trials (portions of fMRI time series) were sorted into the radial condition when the stimulus orientation matched their meridian angles [e.g., vertical (horizontal) Gabors presented in the vertical (horizontal) meridian group] and into the ortho-radial condition when the stimulus orientation is orthogonal to their meridian angles [e.g., vertical (horizontal) Gabors presented in the horizontal (vertical) meridian group]. Regardless of stimulus orientation, all of the trials were categorized into the neutral condition for the oblique meridian group. The radiality analysis was restricted to a population of voxels with eccentricity ranging from $1^{\circ}$ to $6^{\circ}$, where strong and significant $A I_{A X}$ was observed. The remaining parts of the radiality analysis were identical to those of the analysis conducted on the eccentricity bins.

\section{Results}

\section{Coordinates of individual voxels in} visuotopic space

Conventionally, visuotopic coordinates of individual voxels have been estimated by the so-called "phase-encoding" method, in which contrast-defined rings and wedges drifting along the eccentricity and angular axis, respectively, are used to estimate the visuotopic position that generates the greatest fMRI responses for each voxel (Engel et al., 1994; Sereno et al., 1995; Engel et al., 1997). Despite its computational simplicity and efficiency in defining the boundaries between visual areas, the phase encoding method suffers from a failure to map the foveal cortical region and an inaccurate estimation of eccentricity (Dumoulin and Wandell, 2008). To circumvent these limitations, we instead used a model-based method, in which $p R F$ s (Victor et al., 1994) of individual voxels are estimated by fitting a two-dimensional isotropic Gaussian $p R F$ model to fMRI time series, with the assumption of spatiotemporal linearity in fMRI responses (Dumoulin and Wandell, 2008; Kay et al., 2008). In this study, we used the phase-encoding method only to define the boundaries between cortical visual areas (Fig. 1C,D, white lines) and then used the model-based $p R F$ estimation method to define the fine-scale visuotopic coordinates of $p R F$ centers for individual voxels (Fig. $1 A, B$ ). When these Cartesian coordinates of $p R F$ centers were converted into polar coordinates and projected onto the cortical surface (Fig. $1 C, D$, color patches), the distribution of the polar angle and eccentricity coordinates were well matched to the known retinotopic architecture of the human visual cortex (Horton and Hoyt, 1991) and the locations of the meridians that were estimated using the conventional phase-encoding method. We confirmed of the $p R F s$ of the voxels.
B

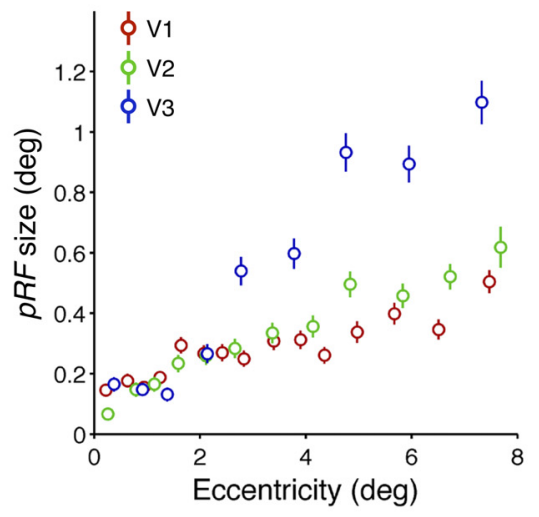

\section{D}

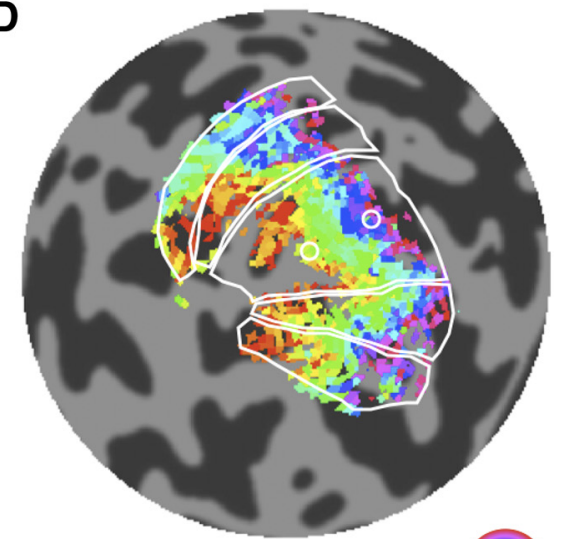

Figure 1. Population receptive fields of the individual voxels in V1, V2, and V3. $\boldsymbol{A}$, Visual field coverage in the central $8^{\circ}$ of the V1 in visuotopic space. $\boldsymbol{B}$, Relationship between eccentricity and $p R F$ size $(\sigma)$ in visuotopic maps of V1, V2, and V3. Each data point

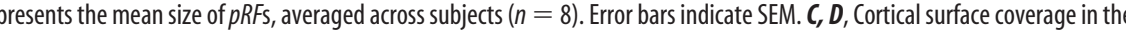
aries. The two small white circles show two example voxels with $p R F$ centers that fall on the horizontal meridian. The corresponding $p R F$ locations of these voxels are indicted by the black stars in $\boldsymbol{A}$. The colors indicate the polar angles $(\boldsymbol{C})$ and the eccentricities $(\boldsymbol{D})$

the validity of our $p R F$ estimation model by also inspecting the relationship between $p R F$ size and eccentricity. Similar to previous fMRI studies (Smith et al., 2001; Kraft et al., 2005; Larsson and Heeger, 2006; Dumoulin and Wandell, 2008), the size of $p R F$ s increased monotonically as a function of the eccentricity of $p R F$ s and across visual areas (Fig. 1B). To further assure the accuracy of the estimated coordinates, we discarded voxels for which the model prediction failed to account for $>25 \%$ of the total variance in fMRI responses to mapping stimuli from further analysis (see Materials and Methods for more details). Even if our $p R F$ estimates are accurate and reliable by themselves, potential errors in coalignment between the $p R F$ mapping session and the experimental sessions could have led to a certain degree of error in estimating the visuotopic coordinates of voxels in the experimental sessions. However, even with the maximum possible size of misalignment (mismatch by a single-voxel size), the resulting amount of errors was $<1^{\circ}$ in the polar angle dimension (mean \pm SE, $0.625 \pm 0.014^{\circ}$ ), which is critical for accurate axial anisotropy estimation (see 
A Session 1: Cardinal orientations
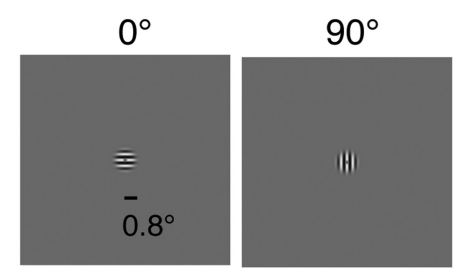

Session 2: Oblique orientations

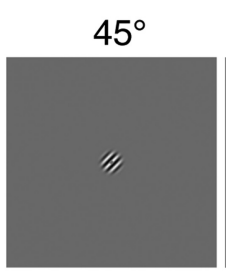

B

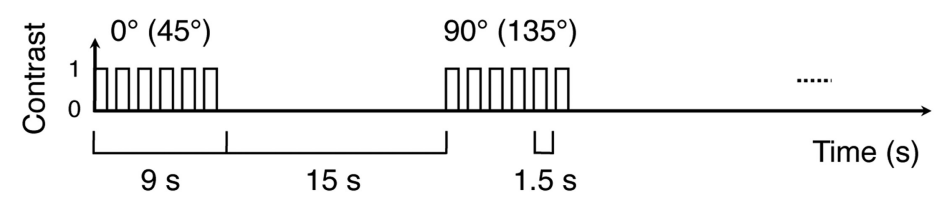

\section{C}

Session 1: Cardinal orientations
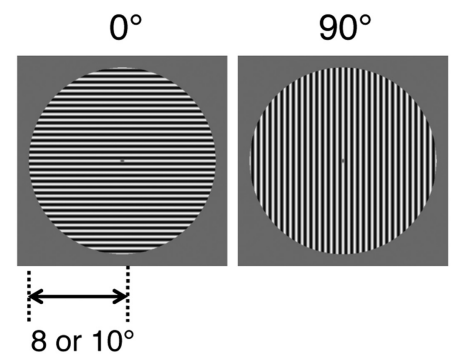

\section{Session 2: Oblique orientations}

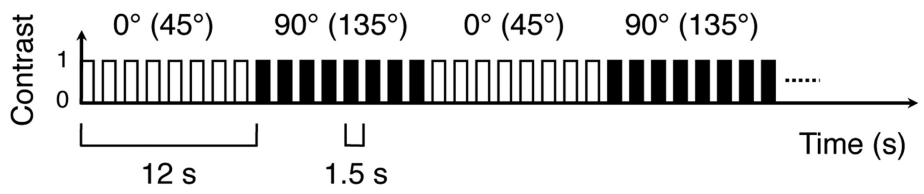

Figure 2. Stimuli and imaging paradigms for the static CPS experiment. A, Trigger stimuli in the CPS triggering runs. A pair of small (radius $=0.8^{\circ}$, indicated by the horizontal bar in the leftmost panel) gratings with cardinal angles was used in one session, and a pair of gratings with oblique angles was in the other session. $\boldsymbol{B}$, Imaging paradigm for the CPS triggering runs. A trigger stimulus flickered at $0.67 \mathrm{~Hz}$ for $9 \mathrm{~s}$ and then turned off for $15 \mathrm{~s}$ before the next trigger stimulus began to flicker. C, Stimuli and fMRI paradigms for the orientation preference mapping runs. Sinusoidal gratings were presented within a large circular (radius $=8.0^{\circ}$ or $10.0^{\circ}$ ) region around the fixation. The orientations of the gratings matched those of the small gratings used in the CPS triggering runs for a given session. The paired gratings alternated every $12 \mathrm{~s}$. The gratings flickered at the same frequency as in the CPS triggering runs.

Materials and Methods for detail), further supporting the robustness of the $p R F$ estimates in our study.

\section{Axial anisotropy of CPS with foveal stimulation}

In the first experiment, with $p R F$ center coordinates defined for individual voxels, we set out to assess the axial anisotropy of CPS by analyzing the pattern of fMRI responses to a small $\left(0.8^{\circ}\right.$ radius $)$ oriented grating (the trigger of CPS) at the fovea (Fig. $2 A$ ) from a population of voxels with $p R F$ centers that covered the visual field far beyond the physical extent of the trigger.

We measured the time series of fMRI responses (Fig. $3 A$ ) to the two orthogonally oriented triggers (Fig. $2 A$ ) in a block design fashion (Fig. 2B). We defined the "peak" of fMRI time series by identifying the time frames (Fig. $3 A$, gray bars) in which the variance across fMRI responses from all voxels within the ROI was maximized (see Materials and Methods for more details). The $P P S F$ was obtained by taking the amplitudes of the peak time series (Fig. 3A, circle symbols) for individual voxels and sorting them as a function of the eccentricity of the $p R F$ centers of voxels (Fig. 3B; see Materials and Methods).

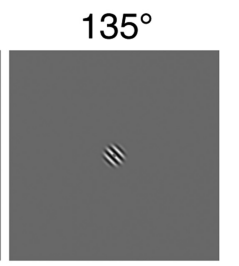

We assessed the axial anisotropy of CPS by comparing two different types of fPSFs: one that is constructed from voxels with $p R F$ centers that were aligned with the orientation axis of the trigger ("coaxial" $f P S F$ ), and the other from voxels with $p R F$ centers that were orthogonal to the axis of the trigger ("ortho-axial" fPSF). For each trial, a given response of a voxel was sorted into the coaxial condition when the absolute offset of its angular position from the trigger orientation was within $22.5^{\circ}$ or into the ortho-axial condition when the offset was $>67.5^{\circ}$. It is important to note that the identity of a given voxel as one of these types of fPSFs was determined not by its absolute $p R F$ location but instead by its location relative to the trigger orientation on a trial-by-trial basis (because trigger orientations varied across trials). For example, an fMRI response from a voxel located in the horizontal meridian (Fig. $1 C, D$, white circles) was sorted into the coaxial condition when the trigger orientation was $0^{\circ}$ or into the ortho-axial condition when the trigger orientation was $90^{\circ}$. This particular aspect of the fMRI experimental design was intended to make the individual voxels contribute evenly to both types of fPSFs over an equal number of trials. This "counterbalance" precluded the possibility that the observed difference between the two types of $f P S F$ s originated by chance from an inherent inhomogeneity of fMRI responses across voxels, possibly because of differences in magnetic susceptibility caused by their geometric locations or differences in signal intensity caused by distances from the receiver coil of the scanner.

Figure $3 A$ contrasts the mean time series of fMRI responses in the coaxial condition (the black lines) to those in the ortho-axial condition (the gray lines) at a few sampled bins of eccentricity in V1 of one representative subject. In both conditions, the fMRI responses around the "peak" time frames (Fig. $3 A$, shaded gray regions) were high and positive in the eccentricity bin close to the foveal region and gradually decreased and became negative with increasing eccentricity. The response amplitude was higher in the coaxial condition than in the ortho-axial condition in the majority of eccentricity bins, indicating that CPS is biased toward the direction that aligns with the trigger orientation.

For quantification and statistical analysis of axial anisotropy, we generated smooth fPSFs by computing the peak amplitudes of fMRI time series averaged within each of the sliding bins of eccentricity (Fig. 3B). Here, the size of the sliding bins was determined to match different eccentricities by cortical distance ( 5 $\mathrm{mm}$ ) based on the cortical magnification factor (Horton and Hoyt, 1991). We examined the axial anisotropy by comparing the spatial extent of positive fMRI responses for coaxial and orthoaxial fPSFs. To define a boundary that split positive and negative lobes of $f P S F$ s while capturing their smooth profiles as a function 
of cortical distance, we adopted the DoG model, which has been used previously to depict the center-surround organization of RFs of V1 simple cells (Hawken and Parker, 1987). We fit the DoG model (Fig. $3 B$, thick smooth curves) to an observed fPSF (Fig. 3B, thin lines) and defined a spatial extent $\left(E_{c}, E_{o}\right)$ by the cortical distance from the stimulus edge at which the fitted DoG model crossed the zero baseline (Fig. 3B, vertical broken lines). Spatial extents were compared between the coaxial and ortho-axial conditions in three visual areas (V1, V2, and V3) from eight subjects (Fig. $3 C$ ).

We indexed the bias of CPS toward the direction aligned with the trigger orientation by computing the difference between the spatial extents from two axial conditions $\left(E_{c}, E_{o}\right)$ relative to the overall spread of activity, which we defined as the axial anisotropy index $\left(A I_{A X}\right.$, Fig. $3 D$; Eq. 6). Despite the individual differences and the small anisotropy indices (median $A I_{A X}=$ 0.02 ; Fig. $3 D$, white arrow in the histogram), the spatial extent was significantly greater in the coaxial $f P S F$ than in the ortho-axial fPSF (Wilcoxon signed rank test, $p=0.003$ ). There was no significant difference between the three visual areas in $A I_{A X}$ (one-way ANOVA, $F_{(2,21)}=$ $0.032, p=0.97)$. Note that the fPSFs were constructed by fMRI responses from the eccentricity bins that have equal sizes in cortical distance with a scale defined by the canonical cortical magnification factor for human visual cortex (Horton and Hoyt, 1991). To compensate for potential inaccuracy of cortical distance estimation because of individual differences in cortical magnification factors, we also estimated $A I_{A X}$ for fPSFs constructed from eccentricity bins that were scaled linearly in visuotopic distance (see Materials and Methods) and confirmed the statistically significant coaxial anisotropy (median $A I_{A X}=0.04$; Wilcoxon signed rank test, $p=$ 0.022 ), without significant differences between the three visual areas in $A I_{A X}$ (one-way ANOVA, $F_{(2,21)}=1.61, p=0.22$ ).

\section{Orientation dependency of axial anisotropy}

Previous anatomical studies demonstrating the coaxial anisotropy of lateral projections in layer 3 of V1 in tree shrews (Bosking et al., 1997) and New World monkeys (Sincich and Blasdel, 2001) also reported that the coaxial lateral connections preferentially occurred among columns with similar orientation preferences. Based on this anatomical evidence for an orientation dependency of coaxial anisotropy, we examined whether the CPS along the coaxial path extended more to the periphery for cortical sites with orientation preferences similar to the orientation of the trigger than those different from the trigger orientation. To this end, we needed to estimate the similarity between orientation preferences of individual voxels and the orientation of the CPS triggers. Two different $(p<0.05)$ than zero.
B

Eccentricity (deg)
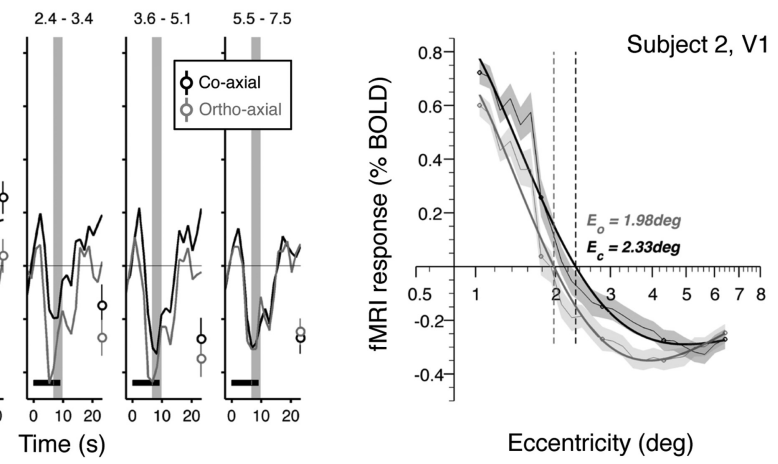

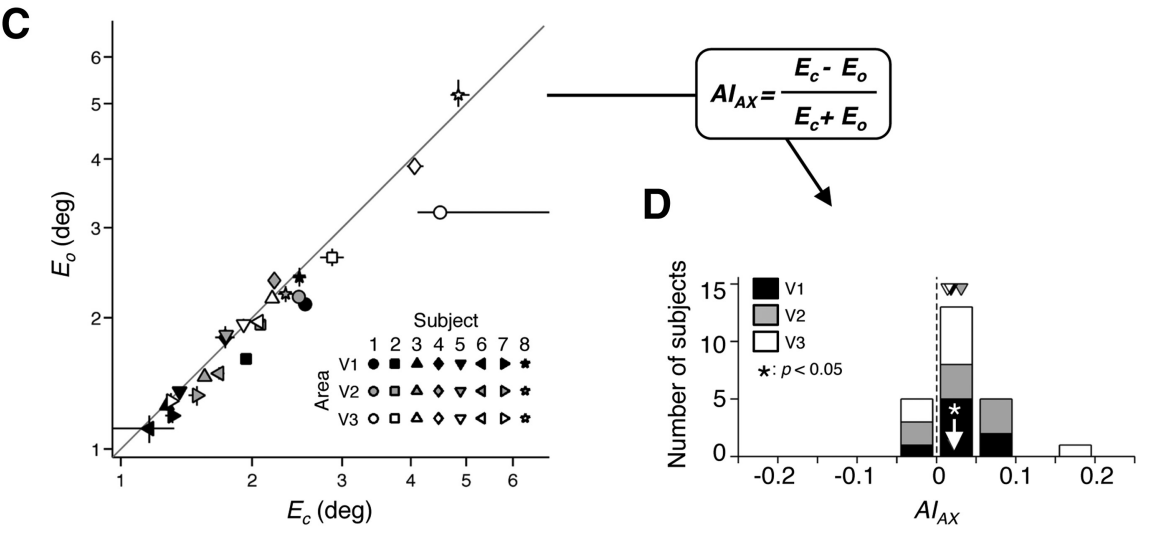

Figure 3. Axial anisotropy in CPS. $A, B$, Example time courses and fPSFs from area V1 of a single subject. $A$, Pairs of averaged time courses of fMRI responses, including one from the coaxial (black) condition and one from the ortho-axial (gray) condition, shown the trial-by-trial distribution of fMRI responses in this zone for each eccentricity bin, the mean amplitudes (the circle lines are the fPSFS, which consisted of sliding-window averages of response amplitudes within eccentricity bins. The translucent patches represent the bootstrap 95\% confidence interval of those averages. The small circles are the average response amplitudes for the eccentricity bins that were sampled in $\boldsymbol{A}$. The thick smooth curves are the DoG models fit to the observed fPSFs. The hyphenated lines indicate the spatial extents of the positive portions of the model fPSFs. The two different brightness levels of the C, D, Population analysis of axial anisotropy. Symbols and brightness levels represent subjects and visual areas, respectively. $C$, triangles of different brightness levels mark the median $A I_{A X}$ values for different visual areas and the white arrow on the $x$-axis marks the grand median $A I_{A X}$ across three visual areas. The asterisk above the arrow indicates that the $A I_{A X}$ values are significantly

orthogonally orientated (either cardinal or oblique) triggers were presented in a given session (Fig. $2 A$ ), so the binary preferences of single voxels were estimated by measuring their fMRI responses to two whole-field gratings with orientations matched to the triggers in the corresponding session (Fig. 2C). We then used the normalized difference between the means of repeated measurements of fMRI responses to those two whole-field gratings as an index for binary orientation preference (Eqs. 7 and 8). The sign and absolute value of the OPI represented the direction and degree of binary preference, respectively. Positive OPIs indicated that fMRI responses of voxels were greater to the trigger orientation than the orthogonal orientation, and OPIs of large absolute sizes indicated big differences in fMRI responses of voxels between the two orientations. The histogram of OPI values for the individual V1 voxels in the coaxial trials from all subjects (Fig. $4 A$ ) indicated that the majority of voxels preferred an orientation aligned with the axis formed by their $p R F$ locations and the fovea. 

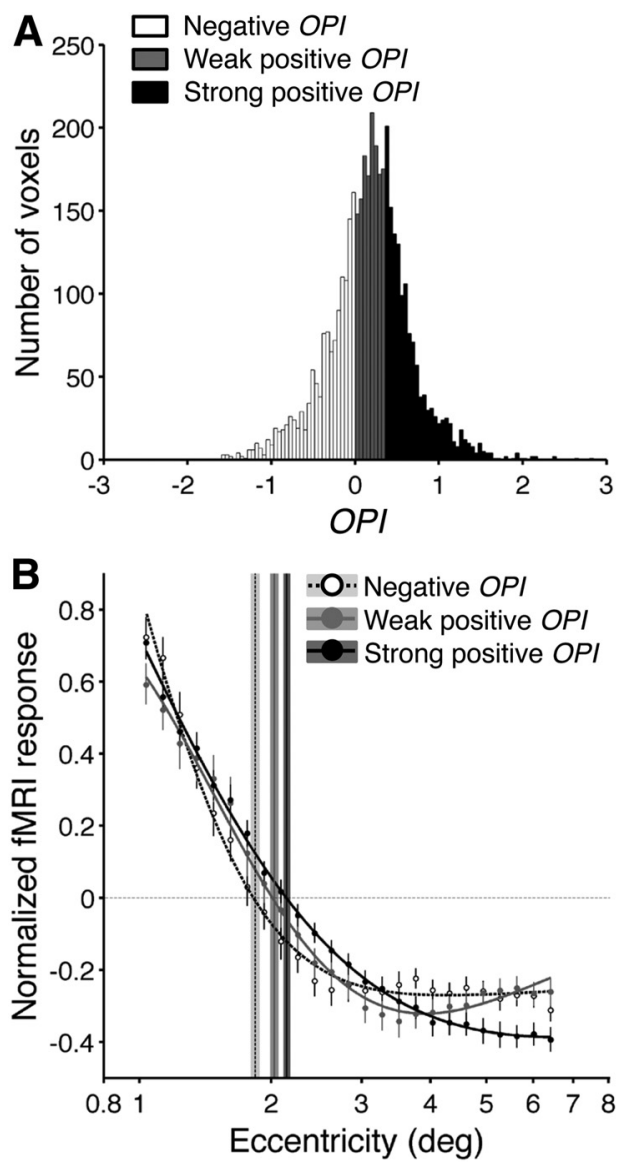

Figure 4. Axial anisotropy dependence on orientation preference of CPS carriers. A, Distribution of the OPIs of entire V1 voxels in the coaxial trials from all subjects. Positive and negative $O P I$ values denote relative preference to orientation (same as and orthogonal to the trigger orientation, respectively). The brightness levels of the bars represent the three groups of voxels with different orientation preferences. $B$, Comparison of fPSFs from the three orientation preference groups. The symbols and error bars around them are the average response amplitudes and the bootstrap $95 \%$ confidence intervals, respectively, at given eccentricity bins. The smooth curves are the DoG models fit to the observed fPSFs. The vertical lines of different colors and styles indicate the spatial extents of those model curves for the three different orientation preference conditions. The widths of shadings around the vertical lines represent bootstrap $95 \%$ confidence intervals.

This positive bias of OPI values was observed in all eight subjects (mean OPI, 0-0.5) and was in good agreement with the so-called "radial bias" of orientation preference, which is a positive correlation between visuotopic angular positions of cortical sites and orientation preferences (Sasaki et al., 2006; Mannion et al., 2010).

To determine whether the spatial extent of CPS along its coaxial path was dependent on the orientation preference of the local sites conveying CPS, we sorted voxels into three different groups based on their OPI values (Fig. 4A, bars in the three different gray levels) and derived $P$ PSFs separately from each of these three groups for comparison (Fig. 4B). Here, the fPSFs were derived from coaxial trials that were merged across all subjects because the number of trials was too small to generate reliable fPSFs from single subjects. Given the overall radial bias in orientation preference, we sorted voxels into "negative OPI," "weak positive $O P I$," and "strong positive OPI" groups. Voxels were assigned to the negative $O P I$ group when their orientation preferences were orthogonal to the orientation of the trigger $(O P I<0$; Fig. $4 A$, white bars). Voxels with preferences that matched the trigger orientation were further divided in half, into the weak (Fig. 4A, gray bars) and strong (Fig. $4 A$, black bars) positive groups. This grouping scheme resulted in an approximately equal number of voxels being assigned to the three different $O P I$ groups (negative OPI group, $32 \%$; weak positive OPI group, $34 \%$; and strong positive OPI group, $34 \%$ ).

The spatial extent of an $P P S F$ along its coaxial path was dependent on the orientation preference of local sites that conveyed the $f P S F$. Specifically, the spatial extent increased as the similarity between the preferred orientations of voxels and the trigger orientation increased (Fig. $4 B$, vertical lines). Both of the two positive $O P I$ groups (Fig. $4 B$, gray and black vertical lines) with orientation preferences that matched the trigger orientation showed spatially more extended $P P S F$ s relative to the negative OPI group (Fig. $4 B$, hyphenated vertical lines) with orientation preferences orthogonal to the trigger orientation (bootstrap test, $p<$ $0.001)$. Moreover, the impact of orientation preference on fPSFs was also evident in the comparison of the two positive OPI groups. The spatial extent of $P P S F$ s was significantly larger in the strong positive OPI group than in the weak positive OPI group (bootstrap test, $p<0.001$ ).

\section{Axial anisotropy only in spatial extension, but not in peak amplitude}

In the first experiment, we observed that CPS was preferentially extended toward cortical sites with $p R F$ locations aligned with the axis of the trigger orientation. Importantly, the trigger varied in orientation but was always presented at a single fixed location in visuotopic space, the fovea. This unifocal stimulation at the fovea allowed us to observe directly coaxial anisotropy in fine-scale spatial profile of CPS (as illustrated in Figs. $3 B$ and $4 B$ ), but also limits the interpretation of the findings in two aspects. First, although a large population of cortical subregions representing the whole visual field under observation participated in shaping CPSs, we do not know whether CPSs exhibit coaxial anisotropy with a similar degree when they are evoked by stimuli presented in the remaining parts of the visual field. Second, a trigger stimulus at the fovea invites uncertainty about the origin of the observed coaxial anisotropy. Previous fMRI studies on human (Sasaki et al., 2006; Mannion et al., 2010; Freeman et al., 2011) or monkey V1 (Sasaki et al., 2006) reported that responses of a given cortical subregion tend to be higher to stimuli with orientation similar to its radial anglular position than to those with dissimilar orienations (dubbed "radial bias"). Because the orientation axis of the foveal stimulus is by definition the radial axis in the visual field, it is possible that the coaxial anisotropy might have reflected the radial bias. However, this possibility is unlikely for several reasons. First, although the CPSs generated by the foveal stimuli in our study reached asymptotic baselines at $\sim 3^{\circ}-4^{\circ}$ (Fig. $4 B$ ) and, more critically, the differences in CPS profile between the two axial conditions occurred in intermediate eccentricities $\left(1.3^{\circ}-3^{\circ}\right)$, the radial bias has been observed reliably in the periphery (Leventhal, 1983; Schall et al., 1986) and failed to be observed in the foveal and intermediate range $\left(<4.5^{\circ}\right)$ of eccentricity (Sasaki et al., 2006; Freeman et al., 2011). Second, in line with this predominance of the radial bias in the periphery, the radial bias is not reliably observed with stimuli with high spatial frequency, even in the periphery (Freeman et al., 2011), whereas stimuli with a high spatial frequency $\left(2 \mathrm{cycles} /{ }^{\circ}\right)$, which was optimized for a cortical population representing the fovea $\left(<0.8^{\circ}\right.$ in eccentricity), were used to evoke CPSs in our study. Third, given its small size in amplitude even when measured via direct stimulation (Sasaki et al., 2006; Mannion et al., 2010; Freeman et al., 2011), the radial bias is unlikely to cause substantial differences in response 
amplitude of cortical population with $p R F s$ located away from the stimulus location. Despite the reasons listed above, however, a potential contribution of the radial bias to the observed coaxial anisotropy cannot be ruled out completely because of the indiscrimination of the radial axis and the orientation axis of stimuli presented at the fovea. To directly address these two issues simultaneously, we conducted the second experiment, in which CPSs were generated throughout a large portion of the visual field by presenting traveling waves of multiple trigger stimuli while varying the traveling direction and the stimulus orientation (Fig. 5A).

In the second experiment, we modified the paradigm used in the $p R F$ mapping experiment by replacing the radial checkerboard stripes with stripes of Gabor patches that were aligned either vertically or horizontally (Fig. 5A). To minimize adaptation and any confounds that may arise because of the relationship between the orientation of the Gabor patches and the direction of the position shift, the relative locations of individual Gabor patches within a stripe were alternated between two positions, and the spatial phases of individual Gabor luminance profiles were altered at every step of the position shift (see Materials and Methods). There were two types of scan runs depending on the global configuration of the Gabor patches, the "vertical stripe" runs (Fig. 5A, top panels) and the "horizontal stripe" runs (Fig. $5 A$, bottom panels). In each cycle of a run, the stripe slowly shifted from one edge to the other edge of the display in one of the directions perpendicular to their global orientation (Fig. $5 \mathrm{~A}$, black arrows). The orientation of local Gabor patches remained unchanged within a cycle but alternated between cycles (Fig. 5A, left and right panels). This orthogonal manipulation of the shifting axis of a global stripe and the orientation of local Gabor patches resulted in four different types of cycles, which were categorized into the "coaxial" condition (Fig. 5A, solid line) or the "orthoaxial" condition (Fig. $5 \mathrm{~A}$, broken line). The rationale for this categorization is as follows. As a local patch slowly approaches the $p R F$ center of a given voxel, the patch would continue to trigger CPS with a center that shifts in phase with the position of the patch along the direction of the shift. As soon as the edge of this dynamic CPS reaches the $p R F$ of that voxel, fMRI responses would depart from the baseline and start increasing. Following this same line of reasoning, fMRI responses of the voxel would remain above the baseline until the other edge of CPS exits the $p R F$ of the voxel. Thus, the time course of the wax and wane of fMRI activity would reflect the spatial profile of CPS that was triggered by the local Gabor patch.

To estimate the spatial extent of CPSs, we analyzed the time courses of fMRI responses in the following steps. First, for each individual voxel, we estimated the time to peak fMRI activity by

A
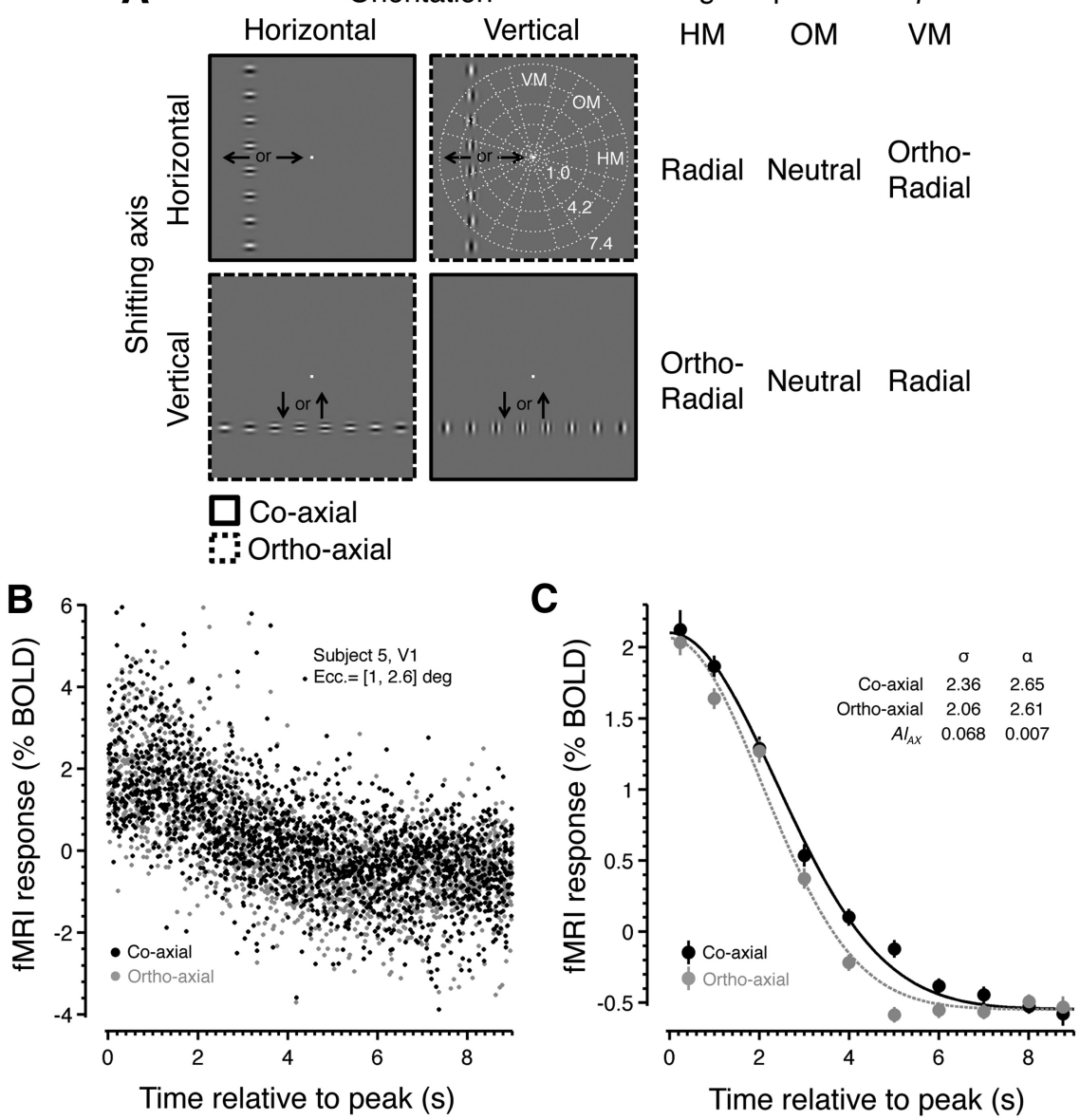

Figure 5. Stimulation conditions and example results of the traveling CPS experiment. $\boldsymbol{A}$, Trial sorting based on the shifting axis and the orientation of triggers. The snapshots of traveling triggers are shown for the four different stimulation conditions, which were the combinations of the two shifting axes (horizontal for the top row and vertical for the bottom row) and the two orientations (horizontal for the left column and vertical for the right column). The black arrows represent a shifting direction of an entire stripe of triggers. The four trigger types were sorted into the coaxial condition (boxed by the black solid lines) or the ortho-axia white dot is the fixation point. The white dotted lines and circles in the top right panel are the boundaries for the angular position groups and the eccentricity bins. The right side of the figure panels shows how the "radiality" conditions are defined based on the conditions, respectively, at one eccentricity bin of a single subject's V1. C Estimation of spatial extent $(\sigma)$ and peak 作 numbers of data points depicted in $\boldsymbol{B}$ are plotted along with the model curves. The axes, symbols, and color labels are the same as in $\boldsymbol{B}$. The inset table in the upper right corner compares the model-fit parameters between the two axial conditions and axial anisotropy indices based on spatial extent and peak amplitude.

fitting cyclic cosine functions to cycle-averaged raw fMRI time series for individual voxels and aligned the time series to the time to peak (see Materials and Methods). Second, we sorted individual voxels into the four eccentricity bins (the boundaries of which are indicated by Fig. $5 \mathrm{~A}$, white dotted iso-eccentricity circles) and further sorted their "time-to-peak-aligned" time series into the coaxial and ortho-axial conditions (Fig. 5B, black and gray dots, respectively). Thus, these merged time series data together constitute a population (aggregate) CPS (pCPS) defined on the time axis for a particular axial condition at a given eccentricity bin. Third, we fitted a Gaussian function to this pCPS and estimated its temporal extent from the width parameter $(\sigma)$ of the fitted Gaussian function (Eq. 10; Fig. 5C). Because a stripe of Gabor patches traveled at a constant speed of $1 \% \mathrm{~s}$, the temporal extent 
A
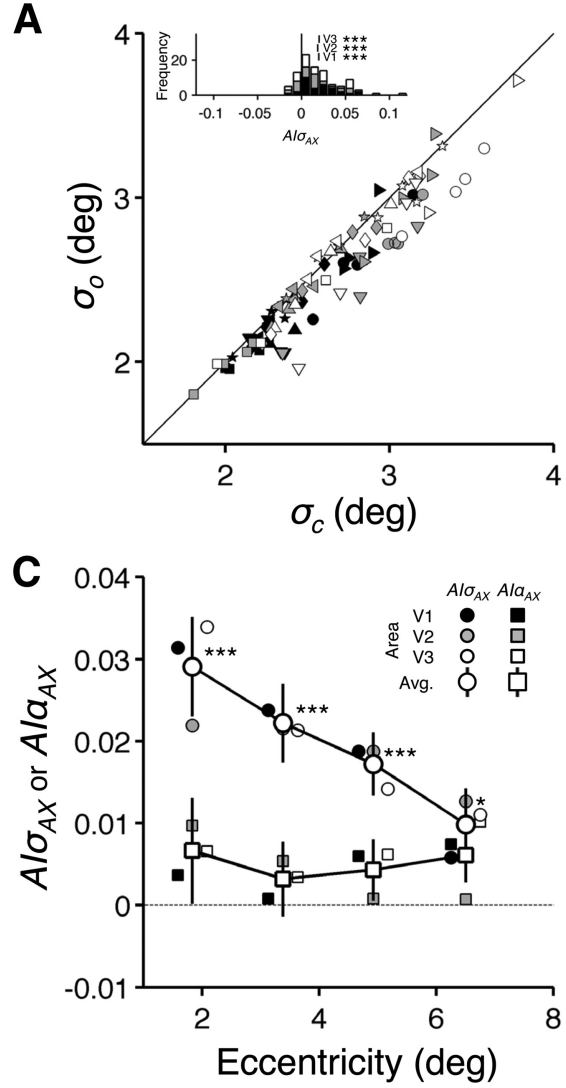

B
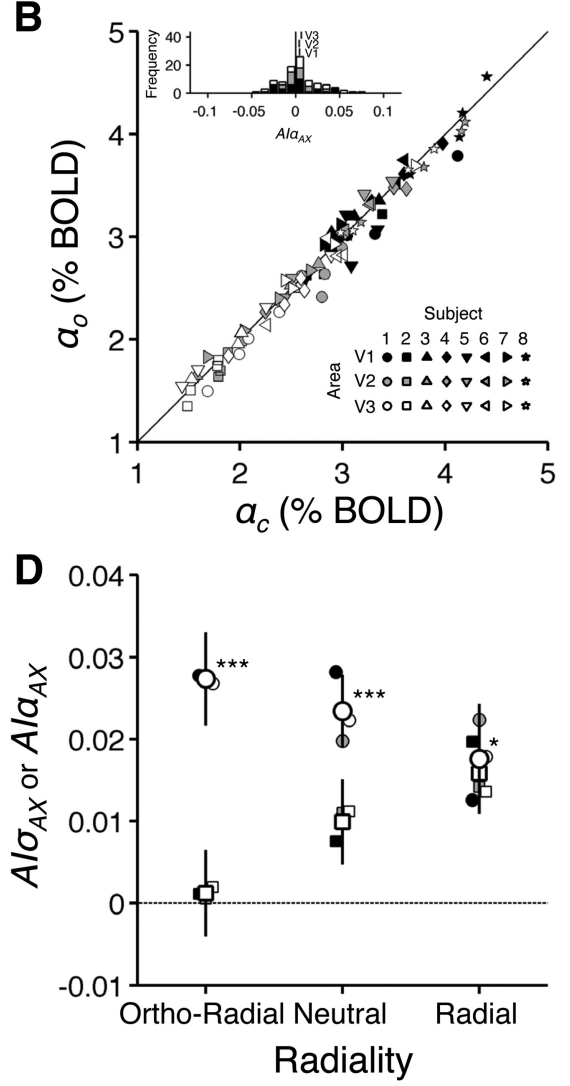

Figure 6. Population analysis of axial anisotropy in the traveling CPS experiment. $\boldsymbol{A}$, Significant coaxial anisotropy in spatial extent of pCPS. The spatial extent estimates in the coaxial condition $\left(\sigma_{c} s\right)$ are plotted against those in the ortho-axial conditions $\left(\sigma_{0} s\right)$ across different eccentricity bins, visual areas, and individuals. Inset (at top), The stacked histograms of $\sigma$-based axial anisotropy indices $\left(A / \sigma_{A X}\right)$, which were computed from the data points below using Eq. 11, separately for different visual areas. The short vertical bars in the inset indicate the averaged $A / \sigma_{A X}$ for different areas. $\boldsymbol{B}$, Absence of coaxial anisotropy in peak amplitude of pCPS. $\boldsymbol{B}$ contains the symbol legends; the format and symbols are the same as in $\boldsymbol{A}$, except that the peak amplitude estimates ( $\alpha_{c} s$ and $\alpha_{0} s$ ) are plotted against each other, and $\alpha$-based axial anisotropy indices ( $\left.A / \alpha_{A X}\right)$ were used for the histograms. $\boldsymbol{C}$, Analysis of eccentricity dependency of axial anisotropy. The $\sigma$-based (circles) and $\alpha$-based (squares) axial anisotropy indices are plotted as a function of eccentricity for different visual areas (black for V1, gray for V2, and white for V3). The large open circles and squares (with error bars) are the averaged $A / \sigma_{A X}$ and $A / \alpha_{A X}$ values, respectively, across different individuals and areas (and the corresponding SEs) at given eccentricity bins. Significance values: ${ }^{*} p<0.05 ;{ }^{* *} p<0.01 ;{ }^{* * *} p<0.001 . \boldsymbol{D}$, Analysis of radiality dependency of axial anisotropy. The format and symbols are the same as in $\mathbf{C}$, except that the anisotropy indices are plotted against the radiality conditions.

can directly translate into the spatial extent. Here, we underline that the $\sigma$ is independent of the amplitude parameter $(\alpha)$ of the fitted Gaussian function, meaning that the $\sigma$ reflects a degree to which a pCPS is spatially extended regardless of an overall amplitude of the pCPS. This independence of $\sigma$ estimates from $\alpha$ estimates ensures that differences in $\sigma$ between a given pair of pCPSs cannot be attributed to any unknown factors that may cause differences in pCPS amplitude. Finally, we computed two types of anisotropy indices: one based on $\sigma$ estimates $\left(A I \sigma_{A X}\right)$ and the other based on $\alpha$ estimates $\left(A I \alpha_{A X}\right)$ using the same formula used in the first experiment (Eq. 11). With these model parameters and anisotropy indices, we compared the two axial conditions in both spatial extent and amplitude of pCPS.

Only the spatial extents, but not the amplitudes, of pCPSs exhibited significant and robust degrees of coaxial anisotropy. When the data in the four eccentricity bins from the eight individual subjects were merged to assess the overall significance of axial anisotropy for a given visual area, the $\sigma$ estimates (Fig. 6A) were significantly larger in the coaxial condition $\left(\sigma_{\mathrm{c}}\right)$ than in the ortho-axial condition $\left(\sigma_{\mathrm{o}}\right)$ for all of the three visual areas $\left(A I \sigma_{A X}\right.$
$=0.020, p<0.0001$ for V1; $A I \sigma_{A X}=$ $0.019, p=0.0002$ for $\mathrm{V} 2 ; A I \sigma_{A X}=0.020$, $p=0.0002$ for V3; paired $t$ test; Fig. $6 A$, inset histograms). In contrast, the amplitude estimates (Fig. 6B, $\alpha_{c}$ and $\alpha_{\mathrm{o}}$ ) did not differ significantly between the two axial conditions in any of the three areas $\left(A I \alpha_{A X}=0.005, p=0.266\right.$ for $\mathrm{V} 1 ; A I \alpha_{A X}$ $=0.004, p=0.292$ for V2; $A I \alpha_{A X}=0.007$, $p=0.112$ for V3; paired $t$ test; Fig. $6 B$, histograms in the inset). This selective manifestation of coaxial anisotropy only in the spatial extent estimates indicates that coaxial stimuli are more effective than ortho-axial stimuli in activating a cortical region that represents a visuotopic region far away from the stimulus, although the two axial types of stimuli are equally effective in driving the activity of a cortical region that represents the stimulus location directly (as illustrated in Fig. $5 C)$. This implies that a potential difference in response amplitude between the two axial conditions, which may arise possibly because of differences in spatial or spatiotemporal second-order features, such as global configuration (among Gabor patches) or apparent motion (between time frames), did not exist, or, even if existed, was too small to affect the spatial extents of pCPSs in our study. On a related note, apparent motion (AM) signals may have created asymmetric shapes of pCPS because motion signals are reported to generate stronger cortical responses in its trailing edge (Whitney et al., 2003; Whitney and Bressler, 2007). According to this scenario, pCPSs with stronger AM would be likely to be more spread in space and weak in amplitude because we merged CPSs that were generated by stimuli traveling in opposite directions. However, our data did not exhibit such a push-pull relationship between the spatial extent and the peak amplitude estimates $(r=-0.004, p=0.950)$, making it unlikely for AM to contribute to the observed coaxial anisotropy.

\section{Axial anisotropy decreasing with increasing eccentricity while} being invariant to radiality

The coaxial anisotropy in spatial extent of pCPS was evident throughout the whole visual filed under observation, and it steadily decreased with an increasing eccentricity. One foremost benefit of the "multifocal CPS" design adopted here is that we can examine how the presence or degree of coaxial anisotropy is dependent on stimulus eccentricity by inspecting the degrees of coaxial anisotropy separately for voxel groups sorted by their $p R F$ eccentricities (Fig. 5A, white dotted concentric circles in the top right panel). When the data were merged across different visual areas and individuals to assess the axial anisotropy at individual eccentricity bins, the $\sigma$-based coaxial anisotropy was significantly larger than 0 at all of the four eccentricity bins $\left(A I \sigma_{A X}=0.029\right.$, $p=0.0001$ for $1^{\circ}<$ eccentricity $\leq 2.6^{\circ} ; A I \sigma_{A X}=0.022, p=$ 0.0001 for $2.6^{\circ}<$ eccentricity $\leq 4.2^{\circ} ; A I \sigma_{A X}=0.017, p=0.0001$ 
for $4.2^{\circ}<$ eccentricity $\leq 5.8^{\circ} ; A I \sigma_{A X}=0.010, p=0.032$ for $5.8^{\circ}$ $<$ eccentricity $\leq 7.4^{\circ}$; paired $t$ tests; Fig. $6 C$, large open circles with error bars and asterisks) and decreased as an increasing function of eccentricity $\left(F_{(3,7)}=2.95, p<0.05\right.$, one-way balanced ANOVA). This reduction of coaxial anisotropy in the periphery cannot be attributed to reduced responses possibly resulting from relatively small stimulus sizes compared with relatively large receptive field sizes in the periphery because there were no differences in peak amplitudes across the eccentricity bins in any visual areas $\left(F_{(3,7)}=0.063, p=0.979, \mathrm{~V} 1 ; F_{(3,7)}=\right.$ $1.098, p=0.367, \mathrm{~V} 2 ; F_{(3,7)}=1.678, p=0.194$, V3; one-way balanced ANOVA, data not shown here). In contrast, the $\alpha$-based coaxial anisotropy values did not differ from zero at any of the four eccentricity bins $\left(A I \alpha_{A X}=0.003-0.007, p=0.072-0.481\right.$; paired $t$ test; Fig. $6 C$, large open squares with error bars) nor demonstrated any differences across the eccentricity bins $\left(F_{(3,7)}=0.12, p=0.947\right.$, one-way balanced ANOVA $)$.

The coaxial anisotropy in spatial extension of pCPSs cannot be explained by the radial bias. Thanks to the orthogonal manipulation of stimulus orientation and radial axis (Fig. $5 A$, columns and rows), we could sort out pCPSs in terms of "radiality" (the similarity of a trigger orientation to the radial axis at a given voxel location; see Fig. 5A, radiality condition table) and then examined whether coaxial anisotropy is dependent on radiality. If the radial bias governs the spatial extents of pCPSs (the "radial bias" hypothesis), the sign and the magnitude of coaxial anisotropy should vary systematically as a function of radiality: a large positive anisotropy in the "radial" condition, a near-to-zero anisotropy in the "neutral" condition, and a large negative anisotropy in the "ortho-radial" condition. However, the $\sigma$-based coaxial anisotropy was robust to the varying degrees of radiality $\left(A I \sigma_{A X}=\right.$ 0.027, $p=0.0001$ for "ortho-radial"; $A I \sigma_{A X}=0.023, p<0.0001$ for "neutral"; $A I \sigma_{A X}=0.018, p=0.013$ for "radial"; Fig. $6 D$, circles). On the other hand, the amplitude estimates of pCPSs confirmed that a significant degree of radial bias indeed existed in our data, as indicated by the significant $A I \alpha_{A X}$ value at the "radial" condition $\left(A I \alpha_{A X}=0.016, p=0.002\right)$, wherein a stimulus with orientation matching a given voxel's angular position happened to be the "coaxial" condition. In summary, pCPSs were preferentially extended farther along the visuotopic axis aligned to the orientation of the trigger regardless of the radial bias, which revealed itself via peak amplitude of pCPSs, rejecting the "radial bias" as an origin of the observed coaxial anisotropy.

\section{Discussion}

We investigated the axial anisotropy of CPS in human visual cortex by analyzing patterns of fMRI responses representing visual space outside the spatial extent of a stimulus that triggers CPS. We first defined $p R F$ s for unit cortical surfaces and then delivered a unifocal stimulation train of oriented gratings at the fovea to drive fPSFs, which are changes in $\mathrm{AMRI}$ response amplitudes as a function of visuotopic distance from the trigger stimulus. When trials were sorted according to coaxiality between trigger orientation and $p R F$, the CPS was anisotropic to the sites with $p R F$ locations that aligned with the trigger orientation. Decomposition of CPS based on the orientation preferences of its units revealed that the coaxial flank of CPS was spatially more extended when carried by cortical sites with orientation preferences similar to the trigger than by those with preferences orthogonal to the trigger. This presence of coaxial anisotropy was further corroborated with a traveling wave experiment, in which periodic waves of CPS that were generated by multifocal triggers traveled across the entire region of the cortical surface that was under observation. The presence of CPSs that are elongated preferentially along the axis aligned to trigger orientation was robust to the varying degree of the radial relationship between the direction of CPS waves and the $p R F$ locations of the cortical sites, denying the radial bias as an origin of the observed coaxial anisotropy. The coaxial anisotropy was also present outside the cortical region representing the fovea, but its degree gradually decreased as the eccentricity of the CPS trigger increased.

\section{Coaxial anisotropy in "stimulus driven" CPS}

Spread of cortical activity beyond classical visual RFs has been observed in various species using diverse measurements with different spatiotemporal resolutions, including intracellular recording (Grinvald et al., 1994; Bringuier et al., 1999), optical imaging with intrinsic (Das and Gilbert, 1995) or voltage-sensitive dyes (Grinvald et al., 1994), and fMRI (Engel et al., 1997; Shmuel et al., 2007). There has been only one previous attempt to directly examine the axial anisotropy of functionally driven point spread of $\mathrm{V} 1$ activity (Chisum et al., 2003). That study inspected the CPS of an intrinsic optical imaging signal to a single oriented stimulus in V1 of anesthetized tree shrews but did not find any significant axial anisotropy. Other than this previous study, single-cell studies on contextual modulation of target stimuli by flankers (Kapadia et al., 1995; Polat et al., 1998; Kinoshita et al., 2009) only indirectly imply the existence of a functional coaxial anisotropy in V1.

The lack of animal studies on functional axial anisotropy is not surprising because the inspection of axial constraints of CPS requires the monitoring of responses from a large population of neurons simultaneously and also definitions of the precise receptive field locations for each individual neuron under observation. Despite the spatiotemporal limit of the sampling unit, fMRI allows the simultaneous acquisition of cortical responses from the entire population in multiple visual areas and the definition of coordinates for individual sites in visuotopic space (Lee et al., 2005, 2007; Dumoulin and Wandell, 2008; Kay et al., 2008). By taking advantage of these features provided by fMRI, we were able to reveal the presence of spatial anisotropy of CPS in human visual cortex. The high sensitivity of fMRI to detect this subtle anisotropy of CPS is consistent with previous reports indicating that a large fraction of fMRI signal reflects subthreshold synaptic and dendritic activity (Viswanathan and Freeman, 2007; Goense and Logothetis, 2008; Kim and Fukuda, 2008).

\section{Neural mechanisms for coaxially anisotropic CPS}

The functional coaxial anisotropy found in this study is consistent with the corresponding anatomical anisotropy found in horizontal connections of animal V1 (Fitzpatrick, 1996; Bosking et al., 1997; Schmidt et al., 1997; Sincich and Blasdel, 2001; Chisum et al., 2003). Our data, which showed an extended CPS along its coaxial path among local sites that shared orientation preferences with the trigger, further corroborate the involvement of horizontal connections, which are also known to be prevalent in neuronal pools that share the same orientation preferences (Bosking et al., 1997; Sincich and Blasdel, 2001). It is worth noting that the architecture of horizontal connections varies substantially across species. The spatial extent of horizontal projections is almost 10 times larger in tree shrew V1 (Fitzpatrick, 1996; Bosking et al., 1997) than in primate V1 (Sincich and Blasdel, 2001; Angelucci et al., 2002). Furthermore, coaxial anisotropy of horizontal connections is prominent in nonprimate V1 (Bosking et al., 1997; Schmidt et al., 1997), whereas horizontal connections in primate V1 exhibit a small (Sincich and Blasdel, 2001) or negligible (Angelucci et al., 2002) degree of anisotropy. If we convert the $A I \sigma_{A X}$ values observed in V1 into anisotropy ratios (ratio of coaxial 
extent to ortho-axial extent), typical anisotropy indices used in anatomical studies, the resulting anisotropy ratios range from 1.01 to 1.15. These values are similar to those in Angelucci et al. (2002) (1.041.3), where the anisotropy in lateral connections of macaque monkeys' V1 was measured in visuotopic scale as in our study.

These correspondences suggest that horizontal connections may be the anatomical origin of the functional anisotropy that we found. However, a comprehensive picture of cortical circuits in V1 hints that horizontal connections are unlikely to be the sole contributor to the coaxially anisotropic CPS. Although interareal connections constitute a very small fraction of total synaptic inputs to V1 neurons (Douglas and Martin, 2004), the feedforward and feedback circuits exert strong functional impacts on responses of V1 neurons to external stimuli, possibly via adaptive gain control (Douglas and Martin, 2007) or selective gating (Gilbert and Sigman, 2007) of intra-areal recurrent circuits. Anatomical studies in New World primates (Angelucci et al., 2003; Shmuel et al., 2005) found that feedback projections from V2 are distributed in an anisotropic pattern within V1 and form an ellipsoidal shape with the axis of elongation matching the preferred orientation axis of the V2 neurons. In addition, the visuotopic extent of feedback projections is increasingly large in proportion to the receptive field size of the projection neurons (Angelucci et al., 2002) and is commensurate with the overall extent of surround suppression of V1 neurons (Mareschal et al., 2001; Cavanaugh et al., 2002). In line with these findings, our data showed that the foveal trigger quenched fMRI responses below baseline (Fig. $3 A$ ) at cortical sites remote $\left(3^{\circ}-8^{\circ}\right.$ in eccentricity) to the CPS origin (Figs. $3 B$ and $4 B$ ). This implies that surround inhibition is an essential component of CPS and indicates a contribution of feedback mechanisms in shaping of CPS, given the correlation between stimulus-evoked negative BOLD signals and neural suppression demonstrated in monkey and human visual cortex (Shmuel et al., 2006; Bressler et al., 2007; Pasley et al., 2007). In this respect, one possible explanation for the absence of functional coaxial anisotropy in Chisum et al. (2003) is that anesthetization of animals might have mitigated the exertion of feedback influences on V1 (Ringach, 2009).

\section{Behavioral correlates of coaxially anisotropic CPS}

Functional characterizations of V1 neurons can be approximated by a set of filters that extract features along a number of stimulus dimensions (Carandini et al., 2005; Lennie and Movshon, 2005). The discovery of anatomical coaxial anisotropy, initially in the horizontal connections in $\mathrm{V} 1$ and more recently in the feedback projections from V2 to V1, provided a principal rule dictating that the architecture of connections between neuronal filters be defined in two distinct spatial feature dimensions: position and orientation. The intriguing constraints of these two primary features on anatomical connections at the earliest cortical stage of visual processing sparked many proposals for linking the anatomical connections among V1 neurons to various types of contextual modulation of visual perception, including contour detection (Polat, 1999), integration of contours (Hess and Field, 1999), visual masking (Polat and Sagi, 2006), visual crowding (Greenwood et al., 2010), apparent speed (Series et al., 2002), illusory contour (Pillow and Rubin, 2002), perceptual filling-in (Polat and Sagi, 2007), and traveling waves of binocular rivalry (Wilson et al., 2001). Our demonstration of coaxially biased spread of cortical activity in human visual cortex provides a crucial link for the anatomical anisotropy seen in visual cortex in animals and the spatial contextual effects seen in human visual perception.
Of particular relevance to this work are psychophysical studies on contour detection and integration, which found that collinear facilitation (enhanced perception of stimuli whose position and orientation share a visuotopic axis) is absent in the periphery (Hess and Dakin, 1997; Williams and Hess, 1998; Zenger-Landolt and Koch, 2001) and inconsistent across studies and human observers (Polat and Sagi, 1994; Williams and Hess, 1998; Shani and Sagi, 2005). In line with these reports, we found that the coaxial anisotropy of CPS gradually decreased as an increasing function of eccentricity. Therefore, future work will be necessary to inspect the correlations between the contextual modulation of "perceptive fields" (Neri and Levi, 2006) or "association fields" (Hess and Field, 1999) and the structure of CPS by capitalizing on individual differences that can be determined using behavioral and cortical data, as exemplified in this study.

\section{References}

Angelucci A, Levitt JB, Walton EJ, Hupe JM, Bullier J, Lund JS (2002) Circuits for local and global signal integration in primary visual cortex. J Neurosci 22:8633-8646. Medline

Angelucci A, Schiessl I, Nowak L, McLoughlin N (2003) Functional specificity of feedforward and feedback connections between primate V1 and V2. Soc Neurosci Abstr 29:911-912.

Bosking WH, Zhang Y, Schofield B, Fitzpatrick D (1997) Orientation selectivity and the arrangement of horizontal connections in tree shrew striate cortex. J Neurosci 17:2112-2127. Medline

Bressler D, Spotswood N, Whitney D (2007) Negative BOLD fMRI response in the visual cortex carries precise stimulus-specific information. PLoS One 2:e410. CrossRef Medline

Bringuier V, Chavane F, Glaeser L, Frégnac Y (1999) Horizontal propagation of visual activity in the synaptic integration field of area 17 neurons. Science 283:695-699. CrossRef Medline

Carandini M, Demb JB, Mante V, Tolhurst DJ, Dan Y, Olshausen BA, Gallant JL, Rust NC (2005) Do we know what the early visual system does? J Neurosci 25:10577-10597. CrossRef Medline

Cavanaugh JR, Bair W, Movshon JA (2002) Nature and interaction of signals from the receptive field center and surround in macaque V1 neurons. J Neurophysiol 88:2530-2546. CrossRef Medline

Chisum HJ, Mooser F, Fitzpatrick D (2003) Emergent properties of layer $2 / 3$ neurons reflect the collinear arrangement of horizontal connections in tree shrew visual cortex. J Neurosci 23:2947-2960. Medline

Dale AM, Fischl B, Sereno MI (1999) Cortical surface-based analysis: I. Segmentation and surface reconstruction. Neuroimage 9:179-194. CrossRef Medline

Das A, Gilbert CD (1995) Long-range horizontal connections and their role in cortical reorganization revealed by optical recording of cat primary visual cortex. Nature 375:780-784. CrossRef Medline

Douglas RJ, Martin KA (2004) Neuronal circuits of the neocortex. Annu Rev Neurosci 27:419-451. CrossRef Medline

Douglas RJ, Martin KA (2007) Recurrent neuronal circuits in the neocortex. Curr Biol 17:R496-R500. CrossRef Medline

Dumoulin SO, Wandell BA (2008) Population receptive field estimates in human visual cortex. Neuroimage 39:647-660. CrossRef Medline

Engel SA, Rumelhart DE, Wandell BA, Lee AT, Glover GH, Chichilnisky EJ, Shadlen MN (1994) fMRI of human visual cortex. Nature 369:525. CrossRef Medline

Engel SA, Glover GH, Wandell BA (1997) Retinotopic organization in human visual cortex and the spatial precision of functional MRI. Cereb Cortex 7:181-192. CrossRef Medline

Fischl B, Sereno MI, Dale AM (1999) Cortical surface-based analysis: II: Inflation, flattening, and a surface-based coordinate system. Neuroimage 9:195-207. CrossRef Medline

Fitzpatrick D (1996) The functional organization of local circuits in visual cortex: insights from the study of tree shrew striate cortex. Cereb Cortex 6:329-341. CrossRef Medline

Freeman J, Brouwer GJ, Heeger DJ, Merriam EP (2011) Orientation decoding depends on maps, not columns. J Neurosci 31:4792-4804. CrossRef Medline

Friston KJ, Williams S, Howard R, Frackowiak RS, Turner R (1996) 
Movement-related effects in fMRI time-series. Magn Reson Med 35:346355. CrossRef Medline

Gilbert CD, Sigman M (2007) Brain states: top-down influences in sensory processing. Neuron 54:677-696. CrossRef Medline

Gilbert CD, Wiesel TN (1983) Clustered intrinsic connections in cat visual cortex. J Neurosci 3:1116-1133. Medline

Girard P, Hupé JM, Bullier J (2001) Feedforward and feedback connections between areas V1 and V2 of the monkey have similar rapid conduction velocities. J Neurophysiol 85:1328-1331. Medline

Glover GH (1999) Deconvolution of impulse response in event-related BOLD fMRI. Neuroimage 9:416-429. CrossRef Medline

Goense JB, Logothetis NK (2008) Neurophysiology of the BOLD fMRI signal in awake monkeys. Curr Biol 18:631-640. CrossRef Medline

Greenwood JA, Bex PJ, Dakin SC (2010) Crowding changes appearance. Curr Biol 20:496-501. CrossRef Medline

Grinvald A, Lieke EE, Frostig RD, Hildesheim R (1994) Cortical pointspread function and long-range lateral interactions revealed by real-time optical imaging of macaque monkey primary visual cortex. J Neurosci 14:2545-2568. Medline

Hawken MJ, Parker AJ (1987) Spatial properties of neurons in the monkey striate cortex. Proc R Soc Lond B Biol Sci 231:251-288. CrossRef Medline

Hess R, Field D (1999) Integration of contours: new insights. Trends Cogn Sci 3:480-486. CrossRef Medline

Hess RF, Dakin SC (1997) Absence of contour linking in peripheral vision. Nature 390:602-604. CrossRef Medline

Horton JC, Hoyt WF (1991) The representation of the visual field in human striate cortex: a revision of the classic Holmes map. Arch Ophthalmol 109:816-824. CrossRef Medline

Jenkinson M, Bannister P, Brady M, Smith S (2002) Improved optimization for the robust and accurate linear registration and motion correction of brain images. Neuroimage 17:825-841. CrossRef Medline

Kapadia MK, Ito M, Gilbert CD, Westheimer G (1995) Improvement in visual sensitivity by changes in local context: parallel studies in human observers and in V1 of alert monkeys. Neuron 15:843-856. CrossRef Medline

Kay KN, Naselaris T, Prenger RJ, Gallant JL (2008) Identifying natural images from human brain activity. Nature 452:352-355. CrossRef Medline

Kim SG, Fukuda M (2008) Lessons from fMRI about mapping cortical columns. Neuroscientist 14:287-299. CrossRef Medline

Kinoshita M, Gilbert CD, Das A (2009) Optical imaging of contextual interactions in V1 of the behaving monkey. J Neurophysiol 102:1930-1944. CrossRef Medline

Kraft A, Schira MM, Hagendorf H, Schmidt S, Olma M, Brandt SA (2005) fMRI localizer technique: efficient acquisition and functional properties of single retinotopic positions in the human visual cortex. Neuroimage 28:453-463. CrossRef Medline

Larsson J, Heeger DJ (2006) Two retinotopic visual areas in human lateral occipital cortex. J Neurosci 26:13128-13142. CrossRef Medline

Lee SH, Blake R, Heeger DJ (2005) Traveling waves of activity in primary visual cortex during binocular rivalry. Nat Neurosci 8:22-23. CrossRef Medline

Lee SH, Blake R, Heeger DJ (2007) Hierarchy of cortical responses underlying binocular rivalry. Nat Neurosci 10:1048-1054. CrossRef Medline

Lennie P, Movshon JA (2005) Coding of color and form in the geniculostriate visual pathway (invited review). J Opt Soc Am A Opt Image Sci Vis 22:2013-2033. CrossRef Medline

Leventhal AG (1983) Relationship between preferred orientation and receptive field position of neurons in cat striate cortex. J Comp Neurol 220:476-483. CrossRef Medline

Mannion DJ, McDonald JS, Clifford CW (2010) Orientation anisotropies in human visual cortex. J Neurophysiol 103:3465-3471. CrossRef Medline

Mareschal I, Sceniak MP, Shapley RM (2001) Contextual influences on orientation discrimination: binding local and global cues. Vision Res 41: 1915-1930. CrossRef Medline

McIlwain JT (1975) Visual receptive fields and their images in superior colliculus of the cat. J Neurophysiol 38:219-230. Medline

Neri P, Levi DM (2006) Receptive versus perceptive fields from the reversecorrelation viewpoint. Vision Res 46:2465-2474. CrossRef Medline

Nestares O, Heeger DJ (2000) Robust multiresolution alignment of MRI brain volumes. Magn Reson Med 43:705-715. CrossRef Medline

Olman CA, Inati S, Heeger DJ (2007) The effect of large veins on spatial localization with GE BOLD at 3 T: displacement, not blurring. Neuroimage 34:1126-1135. CrossRef Medline

Pasley BN, Inglis BA, Freeman RD (2007) Analysis of oxygen metabolism implies a neural origin for the negative BOLD response in human visual cortex. Neuroimage 36:269-276. CrossRef Medline

Pillow J, Rubin N (2002) Perceptual completion across the vertical meridian and the role of early visual cortex. Neuron 33:805-813. CrossRef Medline

Polat U (1999) Functional architecture of long-range perceptual interactions. Spat Vis 12:143-162. CrossRef Medline

Polat U, Sagi D (1994) Spatial interactions in human vision: from near to far via experience-dependent cascades of connections. Proc Natl Acad Sci U S A 91:1206-1209. CrossRef Medline

Polat U, Sagi D (2006) Temporal asymmetry of collinear lateral interactions. Vision Res 46:953-960. CrossRef Medline

Polat U, Sagi D (2007) The relationship between the subjective and objective aspects of visual filling-in. Vision Res 47:2473-2481. CrossRef Medline

Polat U, Mizobe K, Pettet MW, Kasamatsu T, Norcia AM (1998) Collinear stimuli regulate visual responses depending on cell's contrast threshold. Nature 391:580-584. CrossRef Medline

Polimeni JR, Fischl B, Greve DN, Wald LL (2010) Laminar analysis of 7 T BOLD using an imposed spatial activation pattern in human V1. Neuroimage 52:1334-1346. CrossRef Medline

Ringach DL (2009) Spontaneous and driven cortical activity: implications for computation. Curr Opin Neurobiol 19:439-444. CrossRef Medline

Sasaki Y, Rajimehr R, Kim BW, Ekstrom LB, Vanduffel W, Tootell RB (2006) The radial bias: a different slant on visual orientation sensitivity in human and nonhuman primates. Neuron 51:661-670. CrossRef Medline

Schall JD, Vitek DJ, Leventhal AG (1986) Retinal constraints on orientation specificity in cat visual cortex. J Neurosci 6:823-836. Medline

Schmidt KE, Kim DS, Singer W, Bonhoeffer T, Löwel S (1997) Functional specificity of long-range intrinsic and interhemispheric connections in the visual cortex of strabismic cats. J Neurosci 17:5480-5492. Medline

Sereno MI, Dale AM, Reppas JB, Kwong KK, Belliveau JW, Brady TJ, Rosen BR, Tootell RB (1995) Borders of multiple visual areas in humans revealed by functional magnetic resonance imaging. Science 268:889-893. CrossRef Medline

Seriès P, Georges S, Lorenceau J, Frégnac Y (2002) Orientation dependent modulation of apparent speed: a model based on the dynamics of feed-forward and horizontal connectivity in V1 cortex. Vision Res 42:2781-2797. CrossRef Medline

Shani R, Sagi D (2005) Eccentricity effects on lateral interactions. Vision Res 45:2009-2024. CrossRef Medline

Shmuel A, Korman M, Sterkin A, Harel M, Ullman S, Malach R, Grinvald A (2005) Retinotopic axis specificity and selective clustering of feedback projections from V2 to V1 in the owl monkey. J Neurosci 25:2117-2131. CrossRef Medline

Shmuel A, Augath M, Oeltermann A, Logothetis NK (2006) Negative functional MRI response correlates with decreases in neuronal activity in monkey visual area V1. Nat Neurosci 9:569-577. CrossRef Medline

Shmuel A, Yacoub E, Chaimow D, Logothetis NK, Ugurbil K (2007) Spatiotemporal point-spread function of fMRI signal in human gray matter at 7 Tesla. Neuroimage 35:539-552. CrossRef Medline

Silva AC, Lee SP, Iadecola C, Kim SG (2000) Early temporal characteristics of cerebral blood flow and deoxyhemoglobin changes during somatosensory stimulation. J Cerebr Blood Flow Metab 20:201-206. CrossRef Medline

Sincich LC, Blasdel GG (2001) Oriented axon projections in primary visual cortex of the monkey. J Neurosci 21:4416-4426. Medline

Smith AT, Singh KD, Williams AL, Greenlee MW (2001) Estimating receptive field size from fMRI data in human striate and extrastriate visual cortex. Cereb Cortex 11:1182-1190. CrossRef Medline

Victor JD, Purpura K, Katz E, Mao B (1994) Population encoding of spatial frequency, orientation, and color in macaque V1. J Neurophysiol 72: 2151-2166. Medline

Viswanathan A, Freeman RD (2007) Neurometabolic coupling in cerebral cortex reflects synaptic more than spiking activity. Nat Neurosci 10:1308-1312. CrossRef Medline

Whitney D, Bressler DW (2007) Spatially asymmetric response to moving patterns in the visual cortex: re-examining the local sign hypothesis. Vision Res 47:50-59. CrossRef Medline

Whitney D, Goltz HC, Thomas CG, Gati JS, Menon RS, Goodale MA (2003) 
Flexible retinotopy: motion-dependent position coding in the visual cortex. Science 302:878-881. CrossRef Medline

Williams CB, Hess RF (1998) Relationship between facilitation at threshold and suprathreshold contour integration. J Opt Soc Am A Opt Image Sci Vis 15:2046-2051. CrossRef Medline
Wilson HR, Blake R, Lee SH (2001) Dynamics of travelling waves in visual perception. Nature 412:907-910. CrossRef Medline

Zenger-Landolt B, Koch C (2001) Flanker effects in peripheral contrast discrimination-psychophysics and modeling. Vision Res 41:3663-3675. CrossRef Medline 